\title{
Effects of Leading-Edge Vibration on a Spanwise-Tensioned Membrane at Low Reynolds Number
}

\author{
Zheng Zhang ${ }^{\text {al }}$, Andrew Wrist ${ }^{\mathrm{a}}$, and James P. Hubner ${ }^{\mathrm{a}}$ \\ Department of Aerospace Engineering and Mechanics, The University of Alabama, Tuscaloosa, AL, 35487, USA
}

This paper compares the wake characteristics and aerodynamic forces for spanwisetensioned membranes with free and fixed leading edges (LE). LE vibration was generated by placing the membrane within an air flow with a sufficient onset velocity. The flow velocity in the wake of the free or fixed LE membrane was measured using a hot-wire anemometer sensor placed downstream of the trailing edge (TE), and the aerodynamic forces were measured using a pair of load cells. The silicone rubber membrane wings were tested at varying angles-of-attack (-8 to $40^{\circ}$ ), applied strain ( 2 to $8 \%$ ), and a nominal Reynolds number of 55,000. The force results show that the LE vibration increased the lift coefficient but also induced a higher drag penalty, particularly in the pre-stall region, resulting in a lower aerodynamic efficiency than the fixed-LE membranes. As indicated by the hot-wire results, this was due to a considerable broadening of the wake caused by the free LE and a breakdown of the organized fluid-structure interaction and energy spectrum compared to a fixed LE.

\section{Introduction}

Micro air vehicles (MAV) generally operate in the chord Reynolds number $(R e)$ regime below 100,000 (Mueller and DeLaurier, 2003). In this $R e$ regime, the aerodynamic performance of high $R e$-airfoils is affected by laminar boundary layer separation (Laitone, 1997; Lissaman, 1983; Mueller, 1985). For the fixed wing configuration, the

\footnotetext{
1 Corresponding author, E-mail: zzhang23@crimson.ua.edu. Present address: Department of Aerospace Engineering, Embry-Riddle Aeronautical University, Daytona Beach, FL, 32114, USA
} 
presence and size of the separation bubble, or the lack of reattachment, causes a rapid decrease in airfoil performance: i.e., an increase in drag and decrease in lift, thus, deterioration in aerodynamic efficiency (in terms of maximum L/D) (Mueller, 1999).

One of the approaches to eliminate the separation bubble is using leading-edge (LE) devices, such as LE flaps and wires. Jones and Babinsky (2008) installed a static flap and wire in front of the LE of an Eppler E423 wingrather than mounted on the wing surface - to trip the boundary layer at low $\operatorname{Re}(40,000<\operatorname{Re}<120,000)$. They report that the LE flap and wire both improved the lift over the tested angle-of-attack range by eliminating the formation of the laminar separation bubble, while the surface-mounted tape trip was only effective at low angles-of-attack. This research implies that the LE turbulator upstream of the wing is more efficient than the surface-mounted devices.

A biologically-inspired design applicable to both fixed- and flapping-wing MAVs is a flexible membrane-an extensible membrane adhered onto a rigid skeleton (Mueller et al., 2007). A conspicuous phenomenon of a membrane wing is the flow-induced, and at times large-amplitude, membrane vibration or limit cycle oscillation once an onset velocity is achieved. Prior investigations (Albertani et al., 2007; Arbós-Torrent et al., 2013; Hu et al., 2008; Rojratsirikul et al., 2010; Scott et al., 2012; Timpe et al., 2013; Zhang et al., 2014b; Zhang et al., 2015) on membrane wings with a fixed LE and a fixed or free trailing-edge (TE) have demonstrated that the membrane vibration is related to the aerodynamically-induced strain, applied strain, membrane geometry and properties, and boundary configuration. The positive effects of this configuration are an increase in the momentum transfer and a reduction of the time-averaged flow separation over the membrane, thus, increasing lift and delaying the onset of stall. As shown by Scott et al. (2012), the periodic flow features are driven by the membrane tension which is dependent on the material and geometric properties. While the membrane vibration is important in affecting the time-averaged and time-dependent nature of the flow, the unconstrained, scalloped TE—as opposed to an attached, non-vibrating TE—allows momentum and vorticity from the windward side to release earlier into the leeside shear layer, modifying its development and path (Timpe et al., 2013). Optimal sizing of the membrane is a cell aspect ratio of one and aeroelastic parameter $\left(\Pi_{2}\right)$ between 0.5 and 2 (Zhang et al., 2015).

Similar to leading-edge trips or turbulators, of interest in this investigation is if a free (unconstrained) LE will have similar beneficial effects on the flow. Introducing membrane vibration at the LE- the onset location of separation for thin plates - would likely change the formation and evolution of the LE vortex, shear layer and the corresponding wake, possibly leading to a greater potential to passively modify the flow by means of tensioning. A 
similar consideration was investigated by Jaworski (2012) in a study analyzing thrust and aerodynamic forces generated by a plunging/pitching rigid flat plate airfoil using flow potential functions and the Kutta condition. An additional degree of freedom of a sinusoidal oscillation of a LE/TE flap, which behaves similarly to an unconstrained membrane wing LE/TE flutter, was included. Compared to a TE flap, an oscillating LE flap can produce thrust with a smaller portion of the airfoil chord and at a lower frequency, even in a special case when the airfoil is fixed. His study revealed the potential importance of utilizing LE oscillation in not only flapping-wing design but also fixed-wing design.

This investigation presents the effects of LE vibration on wake and aerodynamic forces of a spanwise-tensioned membrane cell with an unconstrained (free) and constrained (fixed) TE in low $R e$ flow $(40,000<\operatorname{Re}<70,000$, most tests were performed at $R e=55,000$ ). The aspect ratio of the membrane cell is unity. To eliminate the effects of the wing-tip vortex, the membrane was attached to spanwise endplates. The global aerodynamic forces on the fixed-free and free-free configurations with varying applied strain and dynamic pressure were measured using a dual load cell setup. The centerline wake aft of the TE was quantified using a single-sensor hot-wire anemometer. The wake velocity deficit, turbulence intensity, and energy spectrum are presented and discussed. The results of this study can also be used to support the numerical simulation in the CFD community for verification and validation purpose. Details of the model material and design are presented in Section 2 and details of the wind tunnel and instrument characteristics are presented in Section 3. Results and discussion are presented in Section 4.

\section{Nomenclature}

$\begin{array}{ll}b & =\text { Membrane cell span, } \mathrm{mm} \\ c & =\text { Membrane cell chord, mm } \\ C_{D} & =\text { Drag coefficient } \\ C_{D, 0} & =\text { Minimum drag coefficient } \\ C_{L} & =\text { Lift coefficient } \\ C_{L, \alpha} & =\text { Lift curve slope, } / \mathrm{rad} \\ E & =\text { Modulus of elasticity, } \mathrm{kPa} \\ f & =\text { Fundamental frequency, } \mathrm{Hz} \\ L / D & =\text { Aerodynamic efficiency }\end{array}$




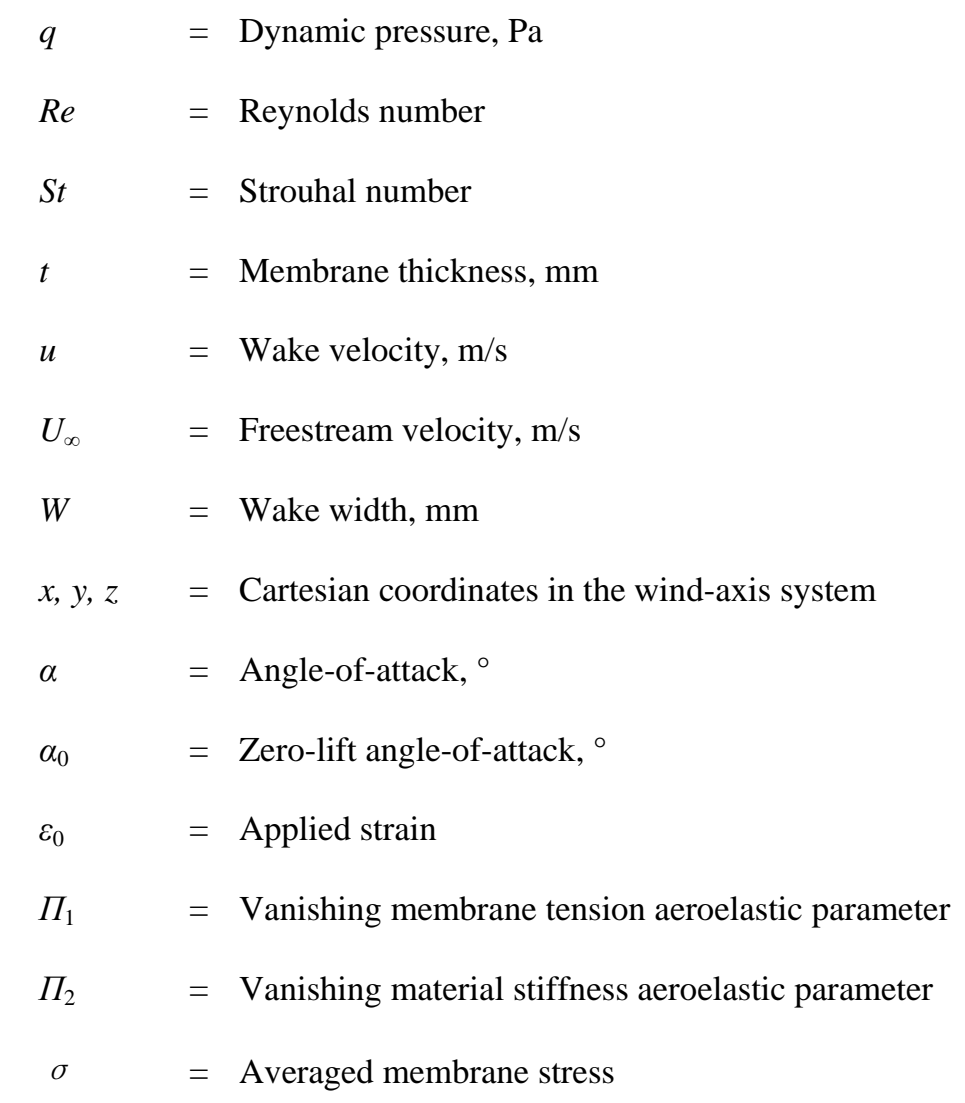

\section{Test Articles}

\subsection{Membrane Cell}

A silicon rubber sheet was used to fabricate the membrane cell. Table 1 lists the measured properties of the silicon rubber.

Table 1. Properties of silicone rubber material

\begin{tabular}{cccc}
\hline $\begin{array}{c}\text { Membrane Thickness } \\
{[\mathrm{mm}] \pm 0.3 \%}\end{array}$ & Modulus of Elasticity & Density $\left[\mathrm{kg} / \mathrm{m}^{3}\right]$ & Hardness $^{1}$ \\
\hline 0.32 & {$[\mathrm{kPa}] \pm 5.5 \%$} & $\pm 1 \%$ & \\
\hline & 450 & 1065 & $20 \mathrm{~A}$ \\
\hline
\end{tabular}

${ }^{1}$ As reported by the manufacturer. 
To construct the spanwise tensioned membrane cell, the silicon rubber sheet was bonded to either two long or short spanwise plates (Fig. 1a and b, respectively) using a procedure described in (Abudaram et al., 2014; Zhang et al., 2014b). The spanwise plates extended to the walls of an insert placed in the wind tunnel (shown in Fig. 1 and further described in Section 3.1). The insert was used to decrease the vertical dimension of the wind tunnel test section and enable shorter spanwise connecting plates. The long attachment plates with a thickness of $2.0 \mathrm{~mm}$ are made of aluminum and the short plates with a thickness of $3.8 \mathrm{~mm}$ are 3D-printed. By nature, silicon rubber has a poor adherence to any surface. To enhance the adhesive ability of the silicon membrane, Primer SS4155 (General Electric) was applied evenly on the desired solid frame surface. When the solvent in the primer was fully evaporated, the adhesive agent (Dragon Skin silicon rubber mixture, Smooth-On) was applied to the target surface of the solid specimen, and the membrane was attached. The excess membrane length was then trimmed after the silicon rubber mixture was cured.

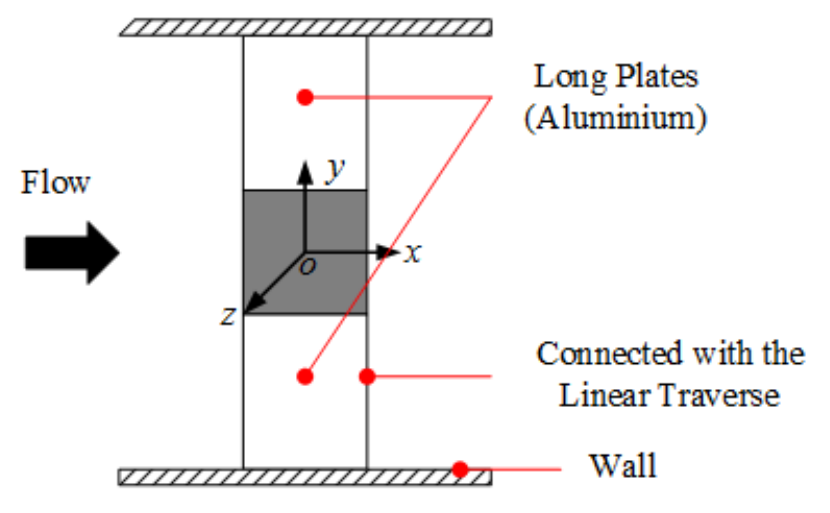

a. Long-plate Setup

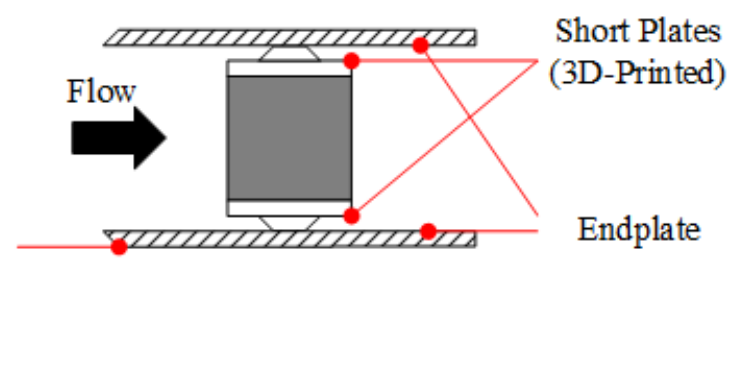

b. Short-plate Setup

Figure 1 Schematic of the spanwise-tensioned membrane cells: silicon rubber (gray) attached to the long (left) and short plates (right). The z-coordinate is into the page.

The existence of the insert walls and endplates effectively eliminated the development of tip vortices. The membrane aspect ratio was one $(76.2 \mathrm{~mm}$ by $76.2 \mathrm{~mm})$ prior to applied tension - a preferred ratio derived from previous investigations on a finite-span, fixed-LE membrane wing (Zhang et al., 2015). The overall initial wall-towall spanwise dimensions of the long-plate (Fig. 1a) and short-plate (Fig. 1b) setups were $30.5 \mathrm{~cm}$ and $8.6 \mathrm{~cm}$, respectively (the latter value varies depending on the membrane spanwise strain, further presented in 3.1). Further dimensions of the set-up are presented in Section 3. 
The spanwise strain in the membrane was created by mechanically stretching the membrane, as described in (Zhang et al., 2014b). The upper plate was fixed to the ceiling of the wind tunnel insert while the lower plate was connected to a linear PC-controlled traverse, which could be positioned at increments of $0.0016 \mathrm{~mm}$. The resolution of the applied strain was $0.002 \%$ based on a span length of $76.2 \mathrm{~mm}$. An in-house LabVIEW program was developed to position the lower plate. Prior to a test, the traverse was manually set at a position of effectively zero strain (except of that induced by the membrane weight, which is significantly lower than the applied strain). The average tensile strain was calculated based on the traversed distance. Pre-strain settings ranged from $2 \%$ to $8 \%$; higher strains induced a substantial contraction in the centerline chordwise dimension of the membrane and, hence, were not examined. Figure 2 compares the applied strain with the optical measured membrane deformation in the spanwise (Fig. 2a) and chordwise (Fig. 2b) direction for the membrane specimen. The optical measured strain was determined by tracking applied targets on the membrane using a digital camera. The nominal strain matched the measured spanwise strain well $\left(R^{2}>0.99\right)$; however, the Poisson effect also existed as shown in Fig. $2 \mathrm{~b}$. As the applied strain reached $8 \%$, a noticeable chordwise shrinking of $4 \%$ developed. Because the LE and TE were free, the chordwise stress at the LE and TE is zero.

The spanwise strain discussed in the current study is a spatially-averaged strain based on the global measurement of the membrane elongation and not the strain field. The nonlinear behaviors of a membrane material like silicone rubber, including geometric nonlinearities (such as finite strain) and material nonlinearities (such as viscoelasticity), are not considered for the estimation of the applied strain. This method reduces computational cost compared to a high-fidelity model, and the outcome is relatively intuitive. A low-fidelity, linear model can characterize the membrane system dynamic behavior effectively well for a moderate to high pre-tensioned (moderately-deformed) membrane which is the case in this study. A high-fidelity, nonlinear model is desired for a low pre-strain membrane (the pre-strain approaching to zero) with a large deformation (Stanford and Ifju, 2008). 


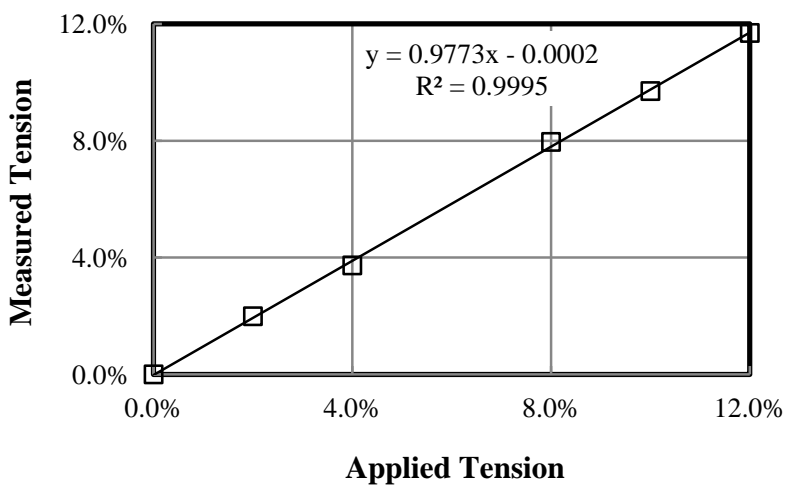

a. Spanwise

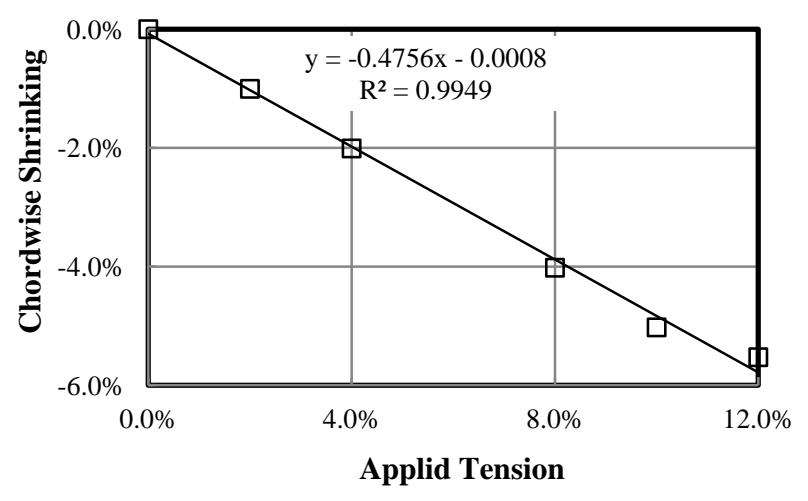

b. Chordwise

Figure 2 Comparison of the desired strain and optically measured strain

Two aeroelastic parameters are usually considered to scale the tension in the membrane relative to the dynamic pressure: the vanishing membrane tension aeroelastic parameter, $\Pi_{1}$, and the vanishing material stiffness parameter, $\Pi_{2}$ (Shyy et al., 2007). In this study, due to the existence of the initial tension, the aeroelastic effect is described by using $\Pi_{2}$. The definition of $\Pi_{2}$ is given by

$$
\Pi_{2}=\frac{\sigma t}{q l},
$$

where $\sigma$ is the averaged membrane stress, $t$ is the membrane thickness, $q$ is the dynamic pressure, and $l$ is the characteristic length. Because the spanwise strain is dominant for a tip-attached membrane, the membrane span length, $b$, is used as the characteristic length. For a dynamic pressure of $70 \mathrm{~Pa}$, the values of the aeroelastic parameter for tensioned membranes of $2 \%, 4 \%$, and $8 \%$ are $0.5,1.1$, and 2.2 , respectively, and includes the elongation in the span due to applied tension. The high value of $\Pi_{2}$ indicates a stiff membrane in the flow, thus, potentially less fluid-structure interaction. As discussed by Zhang et al. (2015), for a $\Pi_{2}>2$, the membrane behaves more similarly as a rigid plate with less aerodynamic benefit from membrane vibration; while for a $\Pi_{2}<0.5$, a strong TE flutter induces higher drag penalty and negative time-averaged effective angle-of-attack, resulting in a poor aerodynamic efficiency and power efficiency. A high-fidelity model is required in this $\Pi_{2}$ range. An optimal $\Pi_{2}$ is between 0.5 and 2.0. 


\subsection{Membrane Constraint}

To explore the effects of the LE vibration on the spanwise tensioned membrane, aerodynamic loads and wake scans were performed. Two types of spanwise constraints were constructed to eliminate the membrane vibration:

1. A pair of U-shaped clips (Fig. 3a) with a chordwise width of $7.6 \mathrm{~mm}, 45^{\circ} \mathrm{LE}$ bevel, and spanwise length of $127 \mathrm{~mm}$ were 3D-printed to constrain the vibration at the LE or TE but allow the spanwise tensioning of the membrane. The overall thickness of the clip was $5.0 \mathrm{~mm}$ and the thickness of the wall was $1.5 \mathrm{~mm}$. The clip was designed to attach to the aluminum plates (gray area in Fig. 3a) via a press fit. A thin foam strip (not shown) was used as filler to eliminate any gap between the membrane (black) and clip (white).

2. A set of thin battens (Fig. 3b), one aluminum and one 3D-printed, with a chordwise width of 3.8 $\mathrm{mm}$ and spanwise length of $212 \mathrm{~mm}$ were fabricated to act as a spanwise batten and constrain displacement away from the LE or TE but allow spanwise tensioning. The thickness of the aluminum batten (gray) is $1.5 \mathrm{~mm}$. The 3D-printed batten (white with a step shown in crosssection view) was $3.5 \mathrm{~mm}$ thick at the middle and $1.5 \mathrm{~mm}$ at the side. The batten set was fixed to the aluminum plates by two screws outside of the membrane portion. The batten was mounted at $25 \%, 50 \%$ or $75 \%$ of the chord. Figure $3 \mathrm{~b}$ shows an example of the batten set installed at the $50 \%$ of the chord. 


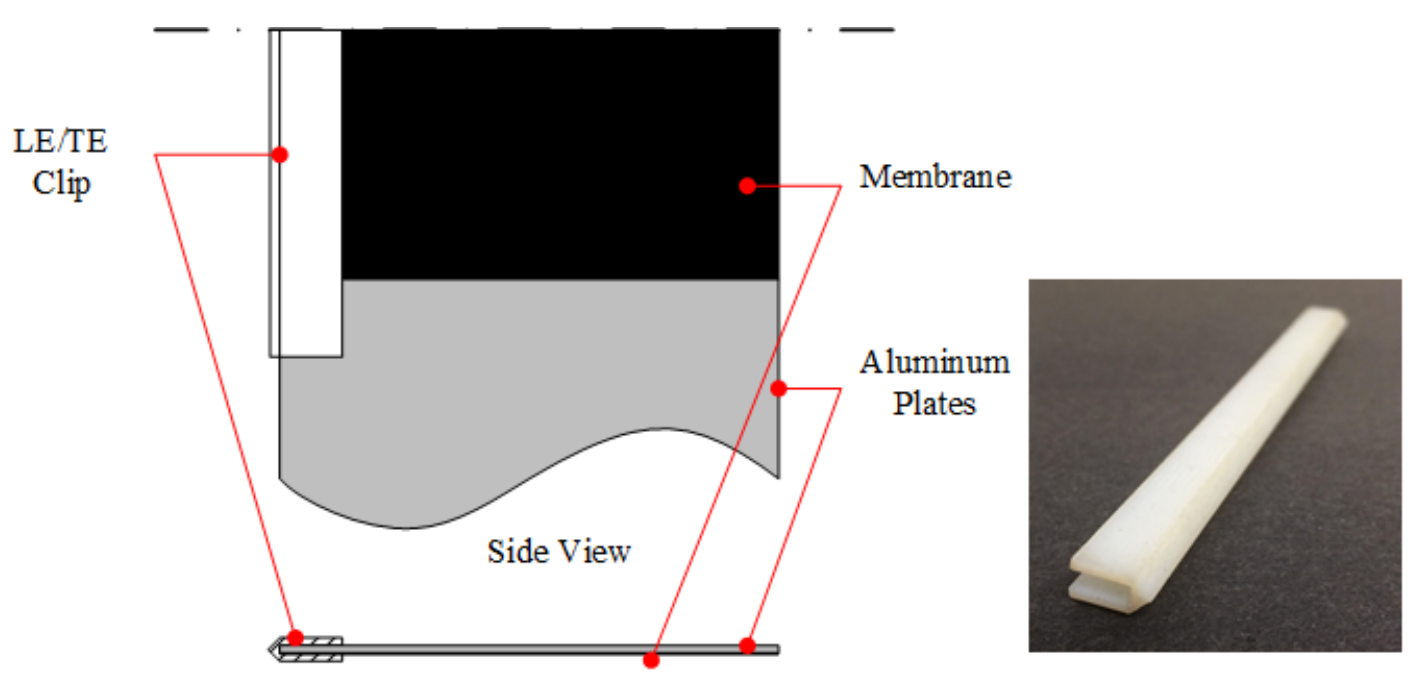

Cross-section View

a. U-shaped clip setup at the LE. The top view and cross-section are presented at left. The right is an image of the

$$
\text { LE/TE clip. }
$$

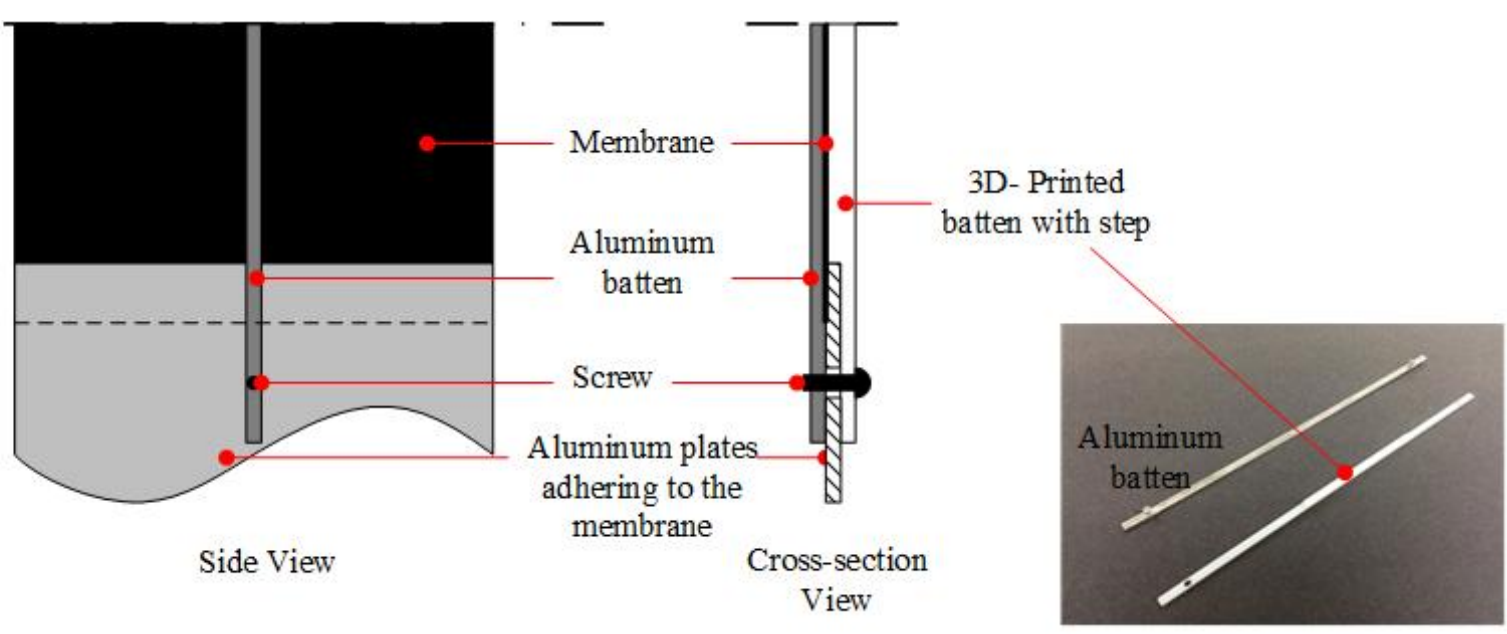

b. Batten setup at the mid-span of the membrane. The left is the top view; the middle is the side view of the setup and the right is an image of the batten.

Figure 3 Membrane constraint at the LE/TE and middle of the membrane; note that for better illustration purpose the drawings shown are not based on actual scale. 
Unavoidably, both clip designs add to the maximum thickness of the model (increase of $t / c$ from $2.6 \%$ to $6.5 \%$ ). However, for thin plates with sharp or square leading edges, separation will occur at the leading edge. As angle-ofattack is increased, the separation grows towards the trailing edge. Except near zero angle-of attack, the clip is in separated flow and the clip thickness is less than the membrane vibration amplitude. Compared to prior force measurement results (Zhang et al., 2015) of a membrane wing model where the membrane was adhered directly to the frame (thus no additional thickness), lift-curve slope measurements are comparable when finite-wing wingtip effects and TE scalloping are considered.

A two-digit nomenclature is used to describe the LE and TE boundary conditions. The first digit indicates the LE condition while the second digit indicates the TE. The designation " 1 " is used for a constrained (or fixed) boundary condition and " 0 " for the unconstrained (or free) condition. For the experiments with the interior batten positioning, the LE and TE were both free. The percentage indicates the batten location from the LE relative to the chord length. The designations and symbols for various membrane configurations for data presentation are summarized in Table 2 .

Table 2. Designations and symbols for the spanwise-tensioned membrane cells

\begin{tabular}{|c|c|c|}
\hline Designation & Symbol & Description \\
\hline $0-0$ & - (red) or $\square$ & free LE - free TE configuration \\
\hline $1-0$ or $0 \%$ & - (green) or $\mathbf{\square}$ & fixed LE - free TE configuration \\
\hline $0-1$ or $100 \%$ & - (blue) or $\mathbf{\square}$ & free LE - fixed TE configuration \\
\hline $1-1$ & - (black) or $\boldsymbol{\square}$ & fixed LE - fixed TE configuration \\
\hline $25 \%$ & - (dark yellow $)$ & batten at $0.25 \mathrm{c}$ from $\mathrm{LE}$ \\
\hline $50 \%$ & $-($ dark red $)$ & batten at $0.50 \mathrm{c}$ from LE \\
\hline $75 \%$ & - (purple) & batten at $0.75 \mathrm{c}$ from LE \\
\hline
\end{tabular}




\section{Experimental Setup}

\subsection{Wind Tunnel}

The experiments were performed in an open-circuit, closed-test section low-speed wind tunnel at the University of Alabama. The flow is drawn into the centrifugal fan through a filtered inlet, discharged through the wide-angle diffuser section, straightened and conditioned by a honeycomb and screen bank, and finally accelerated by the contraction section with a ratio of $4: 1$ before entering the test section. The dimension of the square test section is $0.762 \mathrm{~m} \times 0.762 \mathrm{~m}$. The freestream velocity range is from $1 \mathrm{~m} / \mathrm{s}$ up to $20 \mathrm{~m} / \mathrm{s}$. The walls of the test section are made of clear acrylic panels and glass, providing $360^{\circ}$ optical access.

For the membrane cell tests, the wind tunnel test section was partitioned with an insert to decrease the vertical dimension. This allowed the use of shorter spanwise plates to attach to the membrane. The height between and length (streamwise) of the insert walls for the wake measurements (Fig. 1a) were $30.5 \mathrm{~cm}$ and $61.0 \mathrm{~cm}$, respectively. The initial height between (without applied strain) and length (streamwise) of the insert walls for the force measurement (Fig. 1b) was $8.6 \mathrm{~cm}$ and $22.9 \mathrm{~cm}$, respectively. For the force measurement case, the lower endplate is able to move by means of a PC-controlled traverse which is vertically mounted underneath the floor of the wind tunnel, enabling spanwise tension in the membrane and increasing the spacing between the plates. The leading edges of both inserts were beveled on the outer edge to create a sharp point and flat surface at the entrance. Boundary layer heights are estimated to be $3.0 \mathrm{~mm}$ and $1.6 \mathrm{~mm}$ based on $R e=55,000$ for the wake and force measurement inserts, respectively.

The defined coordinate system is shown in Fig. 1. The $y$-axis is aligned in the spanwise direction passing through the mid-chord of the membrane; thus, the membrane cell rotated about the $y$-axis to change the angle-of-attack. The $x$-axis was aligned in the chordwise direction, and the origin is located at the geometric center of the membrane. The measured freestream turbulence intensity of the test section with the insert (endplates) installed is $0.5 \%$ (without the membrane specimen), indicating that the insert did not have noticeable effect on the flow quality.

\subsection{Wake Velocity Scan}

To detect and measure the wake size and flow fluctuations, a single-sensor hot-wire was scanned across the wake using a constant temperature anemometer (TSI IFA300). The probe (TSI 1210-20), moved by a linear traverse in the $z$-direction, scanned a distance of $230 \mathrm{~mm}$ (three times of the chord length) at $5 \mathrm{~mm}$ increments, thus, 46 
measurements for each wake scan. The sensor was located one chord downstream from the TE and aligned parallel to the TE to be sensitive to the streamwise component of velocity. While the single wire orientation was also sensitive to the vertical component of velocity (in $z$-direction), this component is significantly smaller. The timeresolved PIV measurement on the finite-span membrane wings (Timpe et al., 2013) has shown that the timeaveraged flow reversal is minor at this downstream location. The instantaneous velocity fluctuations, which is represented by $u$, were less than $20 \%$ of the freestream velocity in the tests, as later presented in section 4.1 ; thus, flow reversal in the wake unlikely occurred. The output voltage signal of the anemometer was low-pass filtered at 5 $\mathrm{kHz}$ by an analog filter module and then acquired with an analog input voltage module. Sample frequency was set at $10 \mathrm{kHz}$ for $5 \mathrm{~s}$. The $5 \mathrm{kHz}$ filter setting is approximately 100 times larger than the expected fundamental frequency range of the membrane. A fifth-order polynomial fit calibration was applied to the hot-wire voltage to obtain the instantaneous velocity data. To examine the energy lost caused by wall friction, the freestream velocity was simultaneously monitored by two Pitot-static probes at the entrance of the partitioned test section and at the exit. The difference between the Pitot-static measured freestream velocities at the inlet and outlet of the test section is less than $1 \%$. An in-house LabVIEW program was used to set the probe position, measure the freestream velocity, acquire the voltage data, and apply the calibration. The time-resolved flow velocity was further post-processed to obtain the velocity profile and power spectrum in MATLAB.

Due to the sensitivity of the hot-wire sensor relative to the lab environment and the output signal drift with time, the sensor was calibrated daily using the front Pitot-static probe. The calibration flow speed ranged between $5 \mathrm{~m} / \mathrm{s}$ to $15 \mathrm{~m} / \mathrm{s}$ to meet the expected wake velocity range. A total of eight calibration points were acquired in a random order

to minimize systematic error. After acquiring the calibration data points, the polynomial coefficients were determined using a least-square algorithm.

\subsection{Aerodynamic Force Measurement Setup}

Figure 4 shows the wind tunnel setup for the aerodynamic load measurement on the membrane cell. Two clear Plexiglass endplates were mounted in the test section horizontally to limit the spanwise flow and tip vortex effects. The aerodynamic loads were assessed at the applied strain levels of $2 \%, 4 \%$, and $8 \%$.

The membrane cell was placed vertically in the wind tunnel for both tests. The tips of the membrane were attached to two short spanwise plates (see Figs. 1b and 4), each mounted to a load cell. The 3D-printed short plate was $3.8 \mathrm{~mm}$ wide, $3.8 \mathrm{~mm}$ thick, and $76.2 \mathrm{~mm}$ long; thus the membrane spanwise length was $83.8 \mathrm{~mm}$ under un- 
stretched status. The LE of the short plates were sanded to fit into the slot of the LE/TE clip. The short plates were used for the force measurements to minimize the lift and drag generated by the rigid attachments. Two load cell sensors (Nano-17, ATI Industrial Automation) were used for the force measurements. The load cells were mounted to rotary stages. The gap between the membrane wing and endplate (shown in Fig. 4 right) was designed to be $1 \mathrm{~mm}$, thus, curtailing the gap effect on the test results (Burns and Mueller, 1982). The angle-of-attack was set by manually adjusting the rotary stages, with an accuracy of $\pm 1^{\circ}$. The test angle-of-attack ranged from $-8^{\circ}$ to $40^{\circ}$, at increments of $4^{\circ}$.

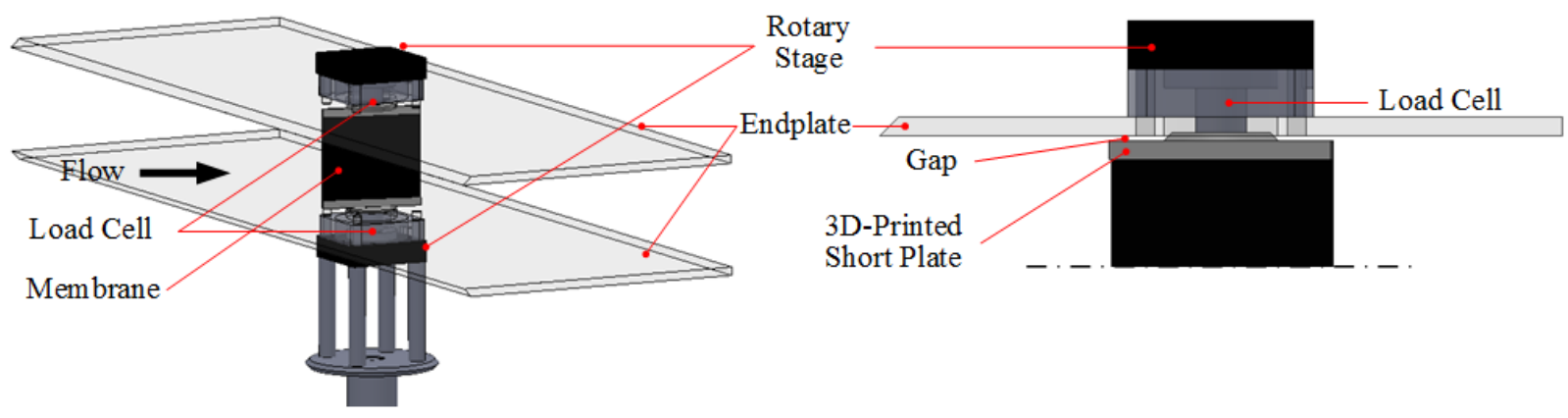

Figure 4 Schematic of the membrane wing setup for the dual-load cell force measurement. The left is the isometric view to show the entire force measurement setup, while the right is a side view to illustrate the gap between the membrane and the top endplate.

The voltage signals of the two load cell sensors were acquired simultaneously by a data acquisition (DAQ) system (SCXI 1520, National Instruments). Each load cell used a dedicated strain gage input module. The voltage signals were sampled at $2 \mathrm{kHz}$ for 10 seconds. During the test, once the angle-of-attack was set, an in-house LabVIEW program was initiated to null the bridge voltage; then, the wind tunnel was powered on and the aerodynamic loads on the membrane were recorded. The membrane was relaxed from the stretched status after testing each day.

To assess the measurement uncertainty, a flat plate was tested twice per day for five consecutive days. The test angle-of-attack ranged from $-8^{\circ}$ to $24^{\circ}$ at $4^{\circ}$ increments. Figure 5 presents the lift coefficients, drag coefficients, and aerodynamic efficiency (in terms of $L / D$ ) with measurement uncertainty level at $95 \%$ confidence interval. The highest uncertainty bound for the lift coefficient occurred at $\pm 4^{\circ}$, which is 0.014 . At high angle-of-attack, the uncertainty level drops to 0.008 . A similar trend was observed in the drag curve plot and the largest uncertainty 
bound is 0.013 at $-8^{\circ}$, thus, resulting in a higher relative uncertainty for aerodynamic efficiency at lower angles-ofattack. Published data by Mueller (1999) with a similar model (flat plate with two endplates) and Reynolds number but a differing LE geometry (this investigation square nose; Mueller elliptic nose) and thicknesses $(2.6 \%$ and $1.9 \%$, respectively) is also plotted as a comparison. Over the test angle-of-attack range, the lift coefficients are effectively the same as the measurements in (Mueller, 1999). Thickness and nose geometry would have minor effects on lift when the flow separates near the LE at non-zero angle of attack. The drag coefficient for the plate investigated here is higher than the comparison data set over the angle-of-attack range, which is likely attributed to the LE geometry, larger relative plate thickness and the presence of the side membrane attachment pieces.
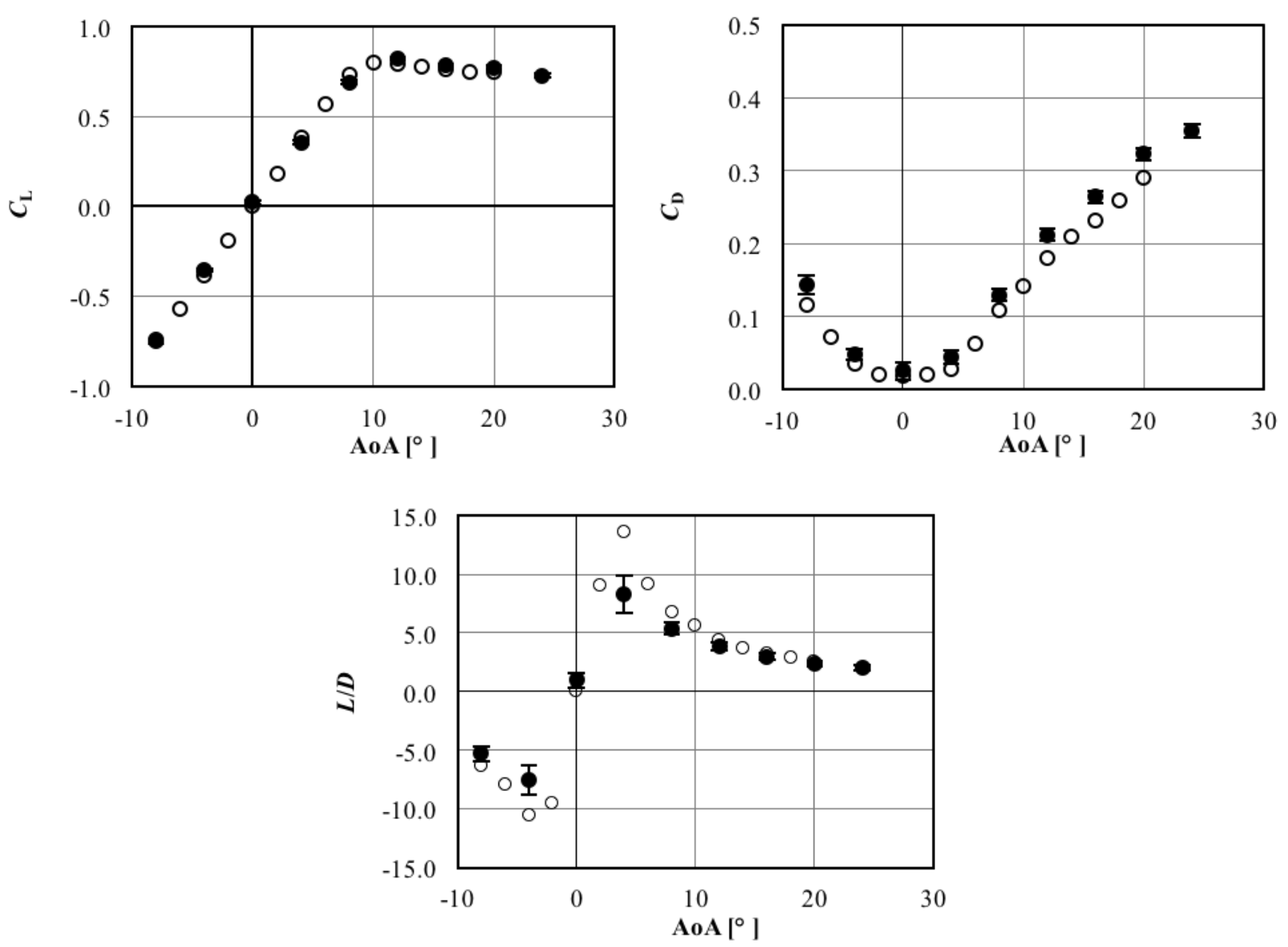

Figure 5 Aerodynamic characteristics with measurement uncertainty level at $95 \%$ confidence interval for the flat airfoil, where the solid symbol $(\bullet)$ represents the measured data $(R e=55,000)$ and the open symbol (○) represents Mueller's data $(\operatorname{Re}=\mathbf{8 0 , 0 0 0})($ Mueller, 1999) 


\section{$4 \quad$ Results and Discussion}

\subsection{Wake Characteristics}

In this section, the wake profiles and spectral energy plots for the spanwise-tensioned membrane are presented and discussed. The corresponding test schematic is shown in Fig. 1a (Section 2.1).

\subsubsection{Data Reduction}

Figure 6 presents a case for a free - free membrane (designated as $0-0$ ) at the test condition of $\alpha=8^{\circ}, \varepsilon_{0}=4 \%$, and $U_{\infty}=10 \mathrm{~m} / \mathrm{s}\left(\operatorname{Re}=55,000\right.$ and $\left.\Pi_{2}=1.1\right)$. The wake velocity is normalized by the freestream velocity, and the probe position is normalized by the chord length. The depth of the wake is defined as the distance from the maximum velocity deficit to the zero velocity deficit. The effect of the wake profile is quantified by the momentum deficit method for incompressible flow:

$$
C_{w}=\frac{2}{c} \sum\left[\left(\frac{u}{U_{\infty}}\right)-\left(\frac{u}{U_{\infty}}\right)^{2}\right] \Delta z
$$

where, $C_{w}$ is defined as wake coefficient, an approximation of the momentum loss.

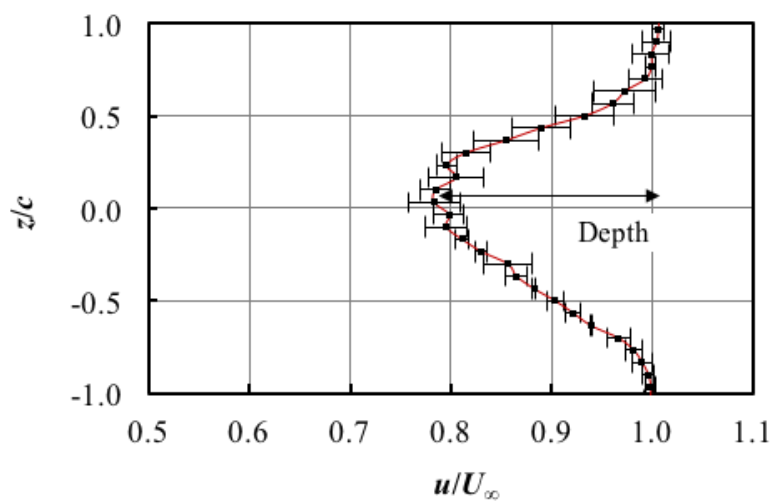

Figure 6 A typical mean velocity profile in the wake with uncertainty level with $95 \%$ confidential interval for the mean velocity profile; the shown case is for the free - free membrane at $\alpha=8^{\circ}, \varepsilon_{0}=4 \%$, and $\Pi_{2}=1.1$. The nondimensional depth is also shown. 
Eq. 2 is an approximation of the profile drag coefficient as it does not consider effects due to the proximity of the probe to the trailing edge, the extent of flow unsteadiness and turbulence behind a vibrating object that may cause instantaneous flow reversal, and the lack of velocity component decomposition. This parameter is presented to qualitatively characterize the net effect of the wake depth and width in Section 4.1.2. It is then compared to the measured drag coefficient by means of a load cell in Section 4.2.2. It is expected that $C_{w}$ will be lower and less accurate than the measured drag coefficient.

A typical measurement uncertainty with $95 \%$ confidence interval for the wake profile and velocity fluctuation (the free - free configuration with $\alpha=8^{\circ}, \varepsilon_{0}=4 \%$, and $\Pi_{2}=1.1$ ) is shown in Fig. 6 as well. The tests were acquired over three consecutive days. The uncertainty bounds are small at the extremes of the wake and relatively high in the center of the wake due to the unsteady, turbulent flow behind the membrane. Overall, the relative measurement uncertainty at $95 \%$ confidential interval based on the sample mean is $1.4 \%$ for both the wake coefficient and nondimensional wake depth.

\subsubsection{Wake Profile}

Figure 7 shows the comparison of the wake mean and root-mean-square (rms) velocity profiles at one chord aft the membrane TE for four boundary conditions (the combination of free and fixed LE/TE). The plate was set at a pre-stall angle-of-attack $\left(\alpha=8^{\circ}\right)$, and the membrane was moderately tensioned at $\varepsilon_{0}=4 \%$. It is apparent that the LE and TE vibration both broadened the wake width compared to the fixed - fixed membrane in the left plot. The membrane with both LE and TE constraints (black) has the narrowest wake width among the four tested specimens. The free - fixed (blue), free - free (red), and fixed - free (green) configurations have a 2.4, 3.4, and 4.1 times larger wake width, respectively, than the fixed - fixed membrane. 

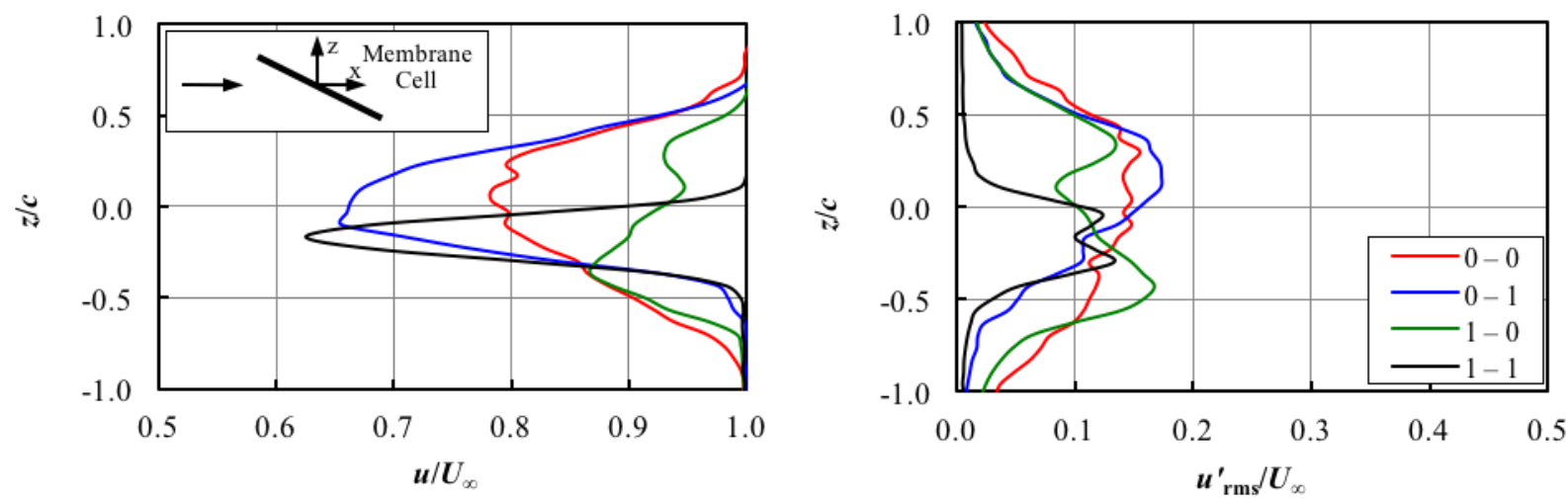

Figure 7 Wake profile (left) and rms fluctuation (right) for the membrane cell with varying boundary conditions $\left(\alpha=8^{\circ}, \varepsilon_{0}=4 \%\right.$, and $\left.\Pi_{2}=1.1\right)$

Another general feature shown in the Fig. 7 is that the depth of the wake is highly dependent on the TE condition. The vibration of a free TE (red and green lines, Fig. 7 left) substantially reduced the maximum velocity deficit and broadened the fluctuation profile as shown by the rms-component (Fig. 7 right). This is an indication of the increased and improved mixing of the higher momentum flow for the free-TE configuration, particularly the windward side release of vorticity at the TE into the wake, as discussed by Timpe et al. (2013).

Due to either a wider or deeper wake velocity profile, the membranes with a free LE have a greater wake coefficient than their fixed-LE counterparts. The fixed - fixed configuration (black), most similar to a rigid plate, exhibited the narrowest and deepest wake profile, the lowest momentum loss, and the smallest flow fluctuation. At $\alpha$ $=8^{\circ}$, the wake coefficient for the free - fixed (blue) and free - free (red) configurations are approximately twice that of the fixed - fixed configuration.

Another noticeable phenomenon in Fig. 7 is a dual-peak in the wake profile plot for the fixed - free configuration (green). The dual-peak exists in all fixed - free cases at lower angles-of-attack $\left(\alpha \leq 12^{\circ}\right.$, as shown in Fig. 8) and different $\operatorname{Re}(40,000 \leq R e \leq 70,000)$ (shown in Zhang et al., 2014a). In addition, this feature is present in the previous study (Timpe et al., 2013) for a finite-span (wing $A R=4.3$ ), fixed-LE membrane wing positioned at $\alpha=$ $4^{\circ}$ and $8^{\circ}$. The top velocity deficit peak (smaller value) is caused by the shear layer emanating from the rigid LE, while the bottom peak (larger value) is related to the shear layer and vortex creation at the TE. The dual peaks are also evident in the rms-velocity plots (Fig. 8, right plot). At the highest angle-of-attack $\left(\alpha=16^{\circ}\right)$, the dual-peak diminishes and the profile resembles that of a typical bluff-body, due to the TE membrane vibration intensifying the 
velocity fluctuation in the wake. The dual-peak feature is more prominent for the higher velocity (lower aeroelastic parameter, i.e. less stiff membrane), suggesting a more pronounced and coherent fluid-structure interaction between the membrane vibration and flow field as well as a steadier and more organized wake structure (as discussed in Section 4.1.3).
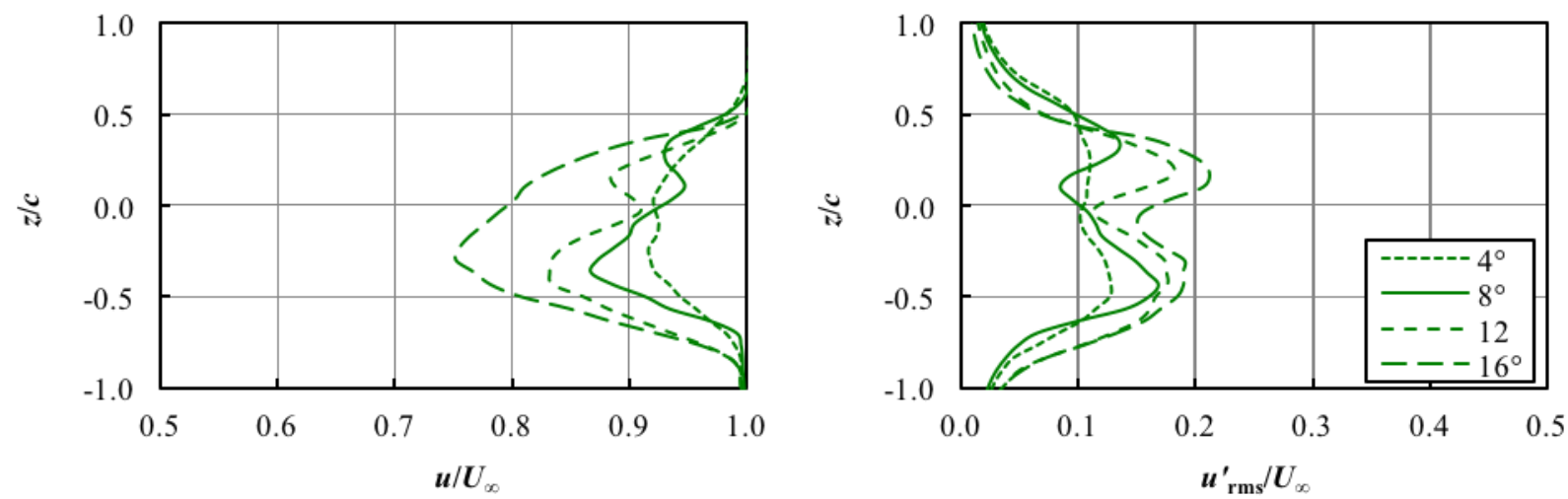

Figure 8 Wake profile and rms fluctuation for the membrane with fixed - free configuration at varying angle-of-attack $\left(\varepsilon_{0}=4 \%\right.$ and $\left.\Pi_{2}=1.1\right)$

Figure 9 compares the wake coefficient, $C_{\mathrm{w}}$, with respect to angle-of-attack for the four combined LE - TE configurations. At all tested angles-of-attack, LE vibration ( $\mathbf{\square}$ and $\square$ ) substantially increased the wake coefficient compared to the fixed-LE configurations. The wake coefficient is related to both the wake width and the wake depth. The wake width represents mixing in the wake, and it was broadened when LE or TE was free. The fixed fixed configuration has the narrowest width (example: Fig. 7, left), thus the least extent of mixing and lowest wake coefficient despite a large wake depth. Once the LE or TE was free to vibrate, the width is relatively independent of the angle-of-attack; however, the width is larger for free TE configurations. The wake depth is primarily affected by the TE constraint. The free-TE configurations show approximately half the deficit than the fixed-TE counterparts due to the periodic release of vorticity into the wake to counter the loss of momentum. While the free - free and free - fixed configurations show the greatest wake coefficients, they are attributed to the different mechanisms. For the free - free membrane, the high wake coefficient is the result of vibrating at both the LE and TE, creating a large width but moderate deficit. For the free - fixed membrane, the greater wake coefficient is the result of the deep wake and slightly smaller width. 


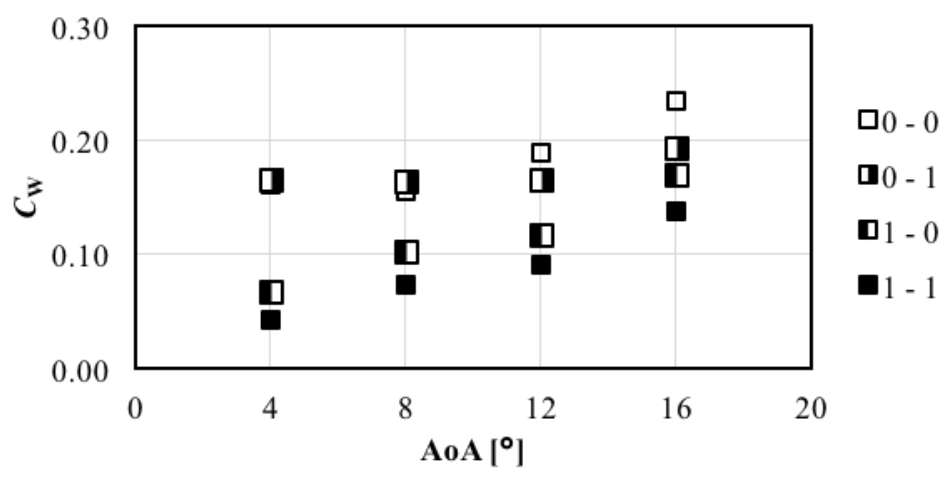

Figure 9 Momentum loss for the membrane cells with varying angle-of-attack at $\varepsilon_{0}=4 \%\left(\Pi_{2}=1.1\right)$

The wake parameter trends are relatively independent of the applied strain for a specific configuration. The wake coefficient trend relative to the applied strain level is shown in Fig. 10 for $\alpha=8^{\circ}$ and $R e=55,000$. The aerodynamic parameter $\Pi_{2}$ values based on the applied strain for the three cases are $0.5,1.1$, and 2.2 , respectively. The wake coefficient for the free - free and fixed - fixed configurations are both relatively insensitive to the change of applied strain level tested; however, the free - free configuration shows a much higher wake coefficient. The applied strain insensitivity was also true for the other configurations (Zhang et al., 2014a). This observation agrees with the effect of membrane pre-strain level on the finite-span membrane wings (Zhang et al., 2015). We report that the pre-strain level does not influence the aerodynamic characteristics as much as the cell and wing geometries. As presented here, both LE and TE vibrations greatly affect the wake coefficient, i.e, the momentum loss that associates with drag. Increasing the applied strain level to the membrane was expected to counter adverse effects of vibration, especially for the free LE case; however, applied tension (up to 8\%) had little effect. 


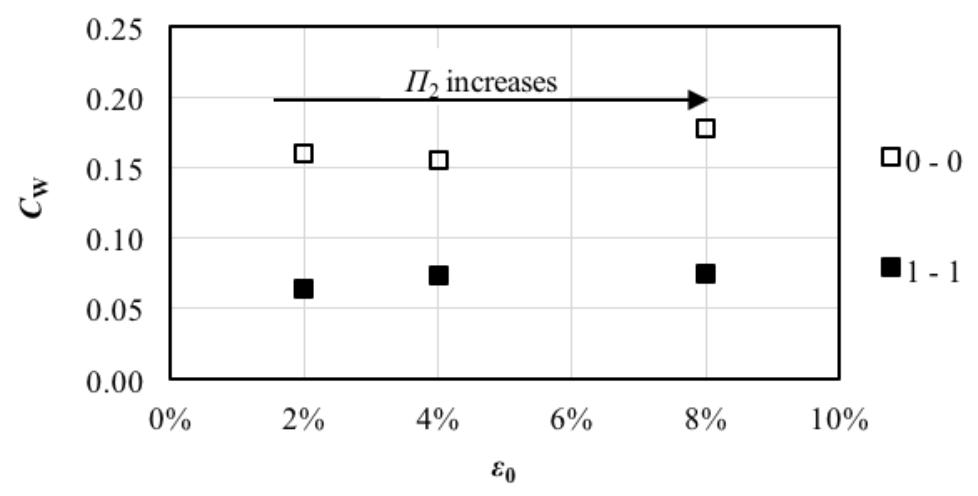

Figure 10 Momentum loss for the membrane cells with varying applied strain at $\alpha=8^{\circ}(\operatorname{Re}=\mathbf{5 5 , 0 0 0})$

To further investigate the effect of LE vibration on the wake profile, the membrane vibration was constrained along the spanwise direction. Using the fixture shown in Fig. 3b, both the LE and TE was free to vibrate. Figure 11 presents the effect of the batten position on the mean velocity profile and velocity fluctuation. The test condition is $\alpha$ $=8^{\circ}, \varepsilon_{0}=4 \%$, and $\Pi_{2}=1.1$. The legend indicates the position of the batten relative to the LE. To better illustrate the LE vibration effect, the fixed - free and free - fixed configurations (previously designated $1-0$ and $0-1$ in Fig. 7) are also plotted, designated as $0 \%$ and $100 \%$, respectively. Clearly visible is that the wake was influenced by the LE vibration more than the chordwise position of the batten. Once the LE vibration was initiated, the wake was relatively independent of the location of the batten, with a similar width and depth in the velocity profile plot as well as a similar turbulence intensity distribution. While not presented, the wake behavior of the batten reinforced membrane cell was also relatively insensitive to the range of the applied strain. 

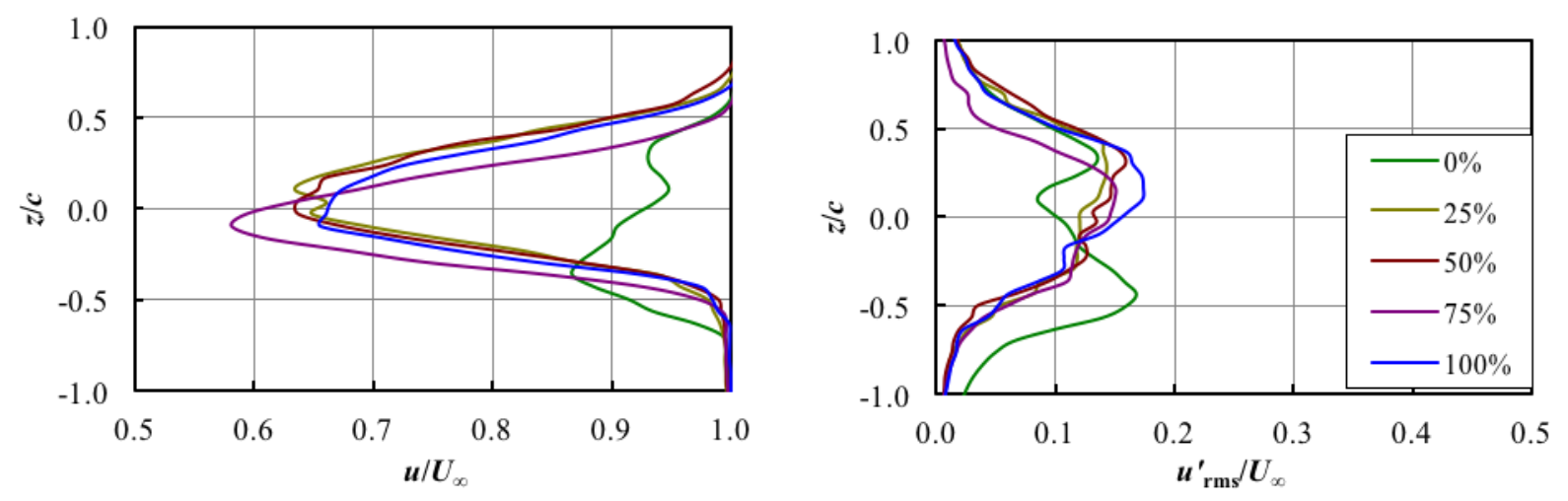

Figure 11 Wake profiles and rms fluctuation for the membrane with varying batten position $\left(\alpha=8^{\circ}, \varepsilon_{0}=4 \%\right.$, and $\left.\Pi_{2}=1.1\right)$

\subsubsection{Spectral Analysis}

In this section, the spectral characteristics of the wake are presented (Figs. 12 and 15) and discussed. The flow fluctuation $\left(u^{\prime}\right)$ power spectra are plotted as waterfall contours across the wake (vertical axis) and over a frequency range between 1 and $200 \mathrm{~Hz}$ (horizontal axis). The grayscale represents the energy magnitude (dark indicates high energy while light indicates low energy). As previously discussed in Section 3, spectral content was captured up to $5000 \mathrm{~Hz}$, but for most cases the dominant fluctuation energy was presented in the range below $200 \mathrm{~Hz}$ (the upper bound of the graphs). To the right of each image, a slice at the peak spectral energy distribution is plotted. The corresponding frequency at the spectral energy peak, $f_{p k}$, is listed below each spectrum.

The effect of membrane boundary conditions (LE and TE) on the power density distribution is presented in Fig. 12. For the free-LE membranes (Figs. 12a and 12c), a fundamental frequency exists but is less organized and with lower peak energy than the fixed-LE membranes (Figs. 12b and 12d). The spectral energy of the free-LE membranes is more disbursed across space and frequency, as indicated by the larger area of gray contours. When the LE vibration is eliminated (Figs. $12 \mathrm{~b}$ and $12 \mathrm{~d}$ ), the energy is concentrated at an easily discernable fundamental frequency and higher harmonics of the membrane. The frequency is a function of the membrane tension, geometry, and properties, and increases with increasing aerodynamically-induced tension (angle-of-attack and dynamic pressure) (Zhang et al., 2014b). The peak spectral energy for the fixed-LE membrane is three times higher than the free-LE counterparts as shown more clearly by the line plots on the right side of each spectral plot. The vibration 
induced at the LE (larger amplitude and more chaotic) distributed energy across a broader range of frequencies. The TE vibration tended to organize both the energy content (as seen by comparing Figs. 12c to 12a, then 12b-the latter displaying higher harmonics) and the fluid-structure interaction between the windward and leeward sides of the membrane. For the fixed-LE configurations (Figs. 12b and 12d), a spatially bimodal distribution forms at the fundamental frequency, consistent with the dual-peak distribution in the rms-velocity profiles presented earlier in Fig. 8 right. This bimodal distribution wanes at higher harmonics. The highly-concentrated energy and organized vibration also corresponds with lower wake deficit and lower drag penalty. Timpe et al. (2013) via time-resolved PIV measurements, showed that a fixed - free configuration resulted in increased momentum transfer induced by the periodic releasing of vorticity from underneath the membrane into the separated region and shear layer. With a free LE, the periodically releasing vorticity into the wake is greatly disrupted and the membrane appears to be less aerodynamically tensioned as indicated by the lower fundamental frequency. Thus, the LE constraint acts as a stiffening agent.

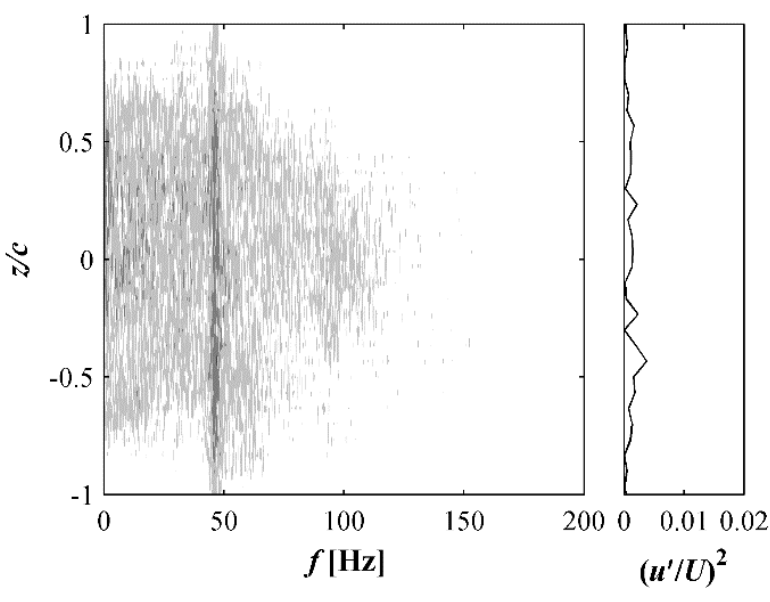

(a) free - free $\left(f_{\mathrm{pk}}=47 \mathrm{~Hz}\right)$

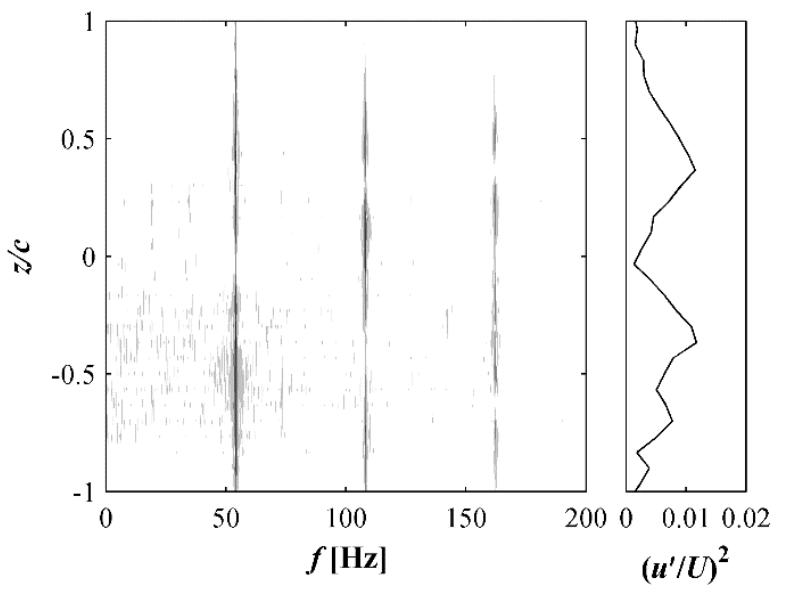

(b) fixed - free $\left(f_{\mathrm{pk}}=54 \mathrm{~Hz}\right)$ 

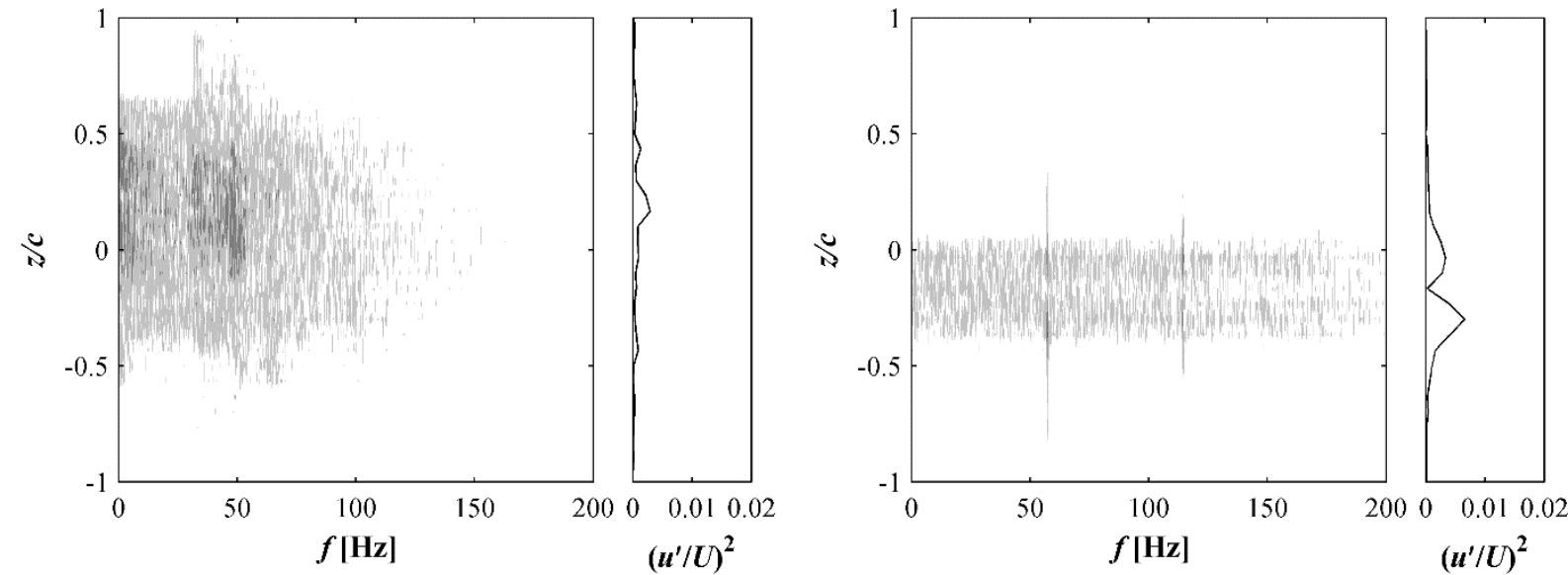

(c) free - fixed $\left(f_{\mathrm{pk}}=49 \mathrm{~Hz}\right)$

(d) fixed - fixed $\left(f_{\mathrm{pk}}=57 \mathrm{~Hz}\right)$

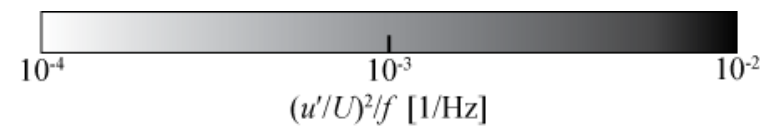

Figure 12 Contour of spectral energy distribution (left) and peak spectral energy (right) for the membrane with varying boundary conditions $\left(\alpha=8^{\circ}, \varepsilon_{0}=4 \%\right.$, and $\left.\Pi_{2}=1.1\right)$

The fundamental frequency trend within the wake with respect to angle-of-attack for the membrane cell is shown in Fig. 13. The frequency trend for the fixed - fixed membrane (-) is relatively linear with increasing angle-ofattack, due to increasing aerodynamic tensioning. However, the free - free membrane ( $\square$ ) shows an $\alpha$-independent fundamental frequency, indicating that the free LE disrupts the additional aerodynamic tensioning with angle-ofattack and increased lift. The projected-chord $S t$ for the fixed - fixed configuration ( $\mathbf{-})$ reached 0.14 at the highest angle-of-attack, providing further evidence that the free LE configuration acts more like a bluff-body (generally occurs around projected-chord $S t=0.15$ and 0.22 ). 


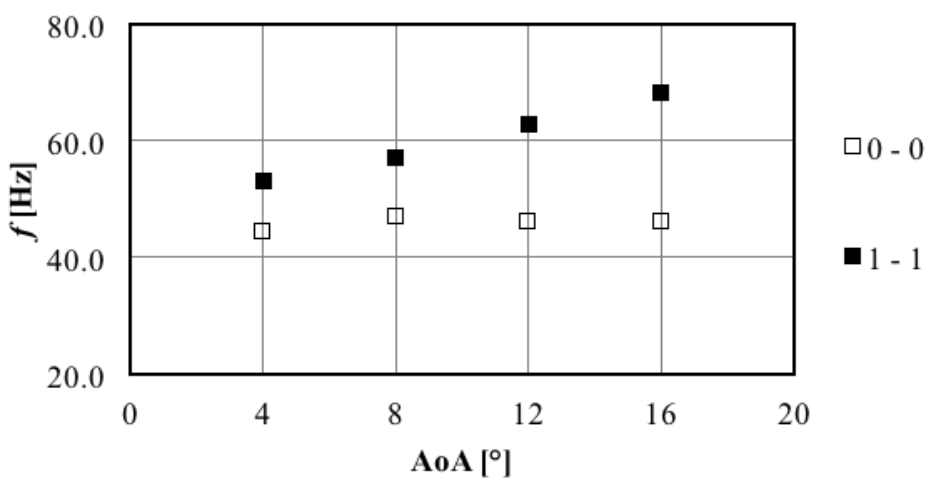

Figure 13 Frequency versus angle-of-attack for the membrane with varying boundary condition $\left(\varepsilon_{0}=4 \%\right.$ and

$$
\left.\Pi_{2}=1.1\right)
$$

Furthermore, Strouhal number (St) based on the wake fundamental frequency for the membranes (Eq. 3) with various LE/TE boundary conditions is presented in Fig. 14:

$$
S t=f W / U_{\infty}
$$

where the width of the wake, $W$, was used as the characteristic length and $U_{\infty}$ is the freestream speed. The test conditions are the same as Fig. 9. At the lower angles-of-attack, the free-LE or TE vibration induced a higher $S t$ compared to the fixed - fixed membrane due to the wider wake as shown in the wake spectrum (Fig. 12). However, at the higher angles-of-attack $\left(12^{\circ}\right.$ and $\left.16^{\circ}\right)$, the difference between the free and fixed boundaries (i.e. LE and TE) decreases. This is due to the increased frequency for the fixed - fixed as evidenced in Fig. 13. The overall $S t$ range of the wake structure is between 0.40 and 0.80. Triantafyllou et al. (1991) pointed out that a preferred $S t$ for thrust production is between 0.25 and 0.35 . In this study, the wake resonance has no contribution to the efficient thrust production; namely, the membrane-vibration-induced coherent wake structure does not show positive effect on the drag reduction. The only case with $S t$ in the preferred thrust generation range is the fixed - fixed membrane at $4^{\circ}$ which is 0.23 . The fixed - fixed membrane did exhibit a lowest drag coefficient as further shown in 4.2.2. It implies that for the consideration of drag reduction, lower amplitude oscillation and lower frequency of the membrane is desired. 


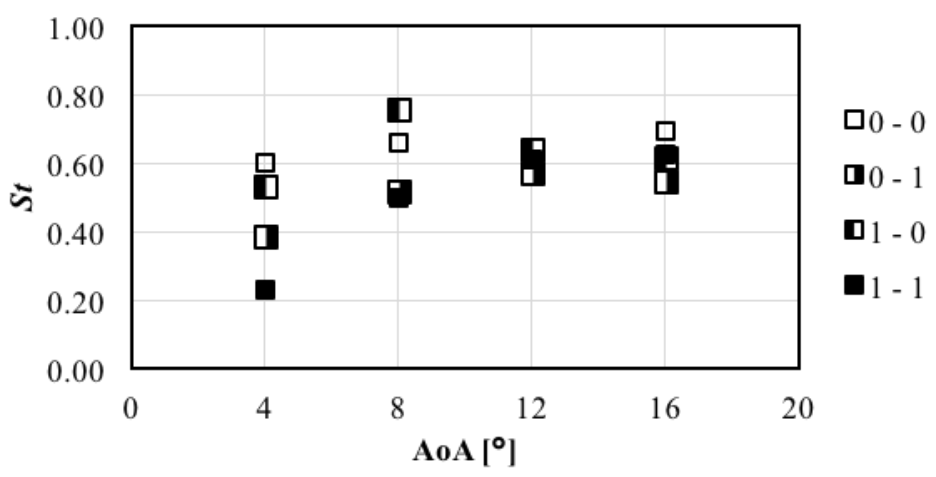

Figure 14 Strouhal number versus angle-of-attack for the membrane with varying boundary condition $\left(\varepsilon_{0}=\right.$ $4 \%$ and $\left.\Pi_{2}=1.1\right)$

Figure 15 presents a series of spectral energy contour plots for membranes with varying batten position: parallel to the LE at $25 \%, 50 \%$ and $75 \%$ of the chord, respectively. The graphs in the left column present energy spectra for the $4 \%$-tensioned membrane and the right column the $8 \%$-tensioned membrane. As discussed previously, the position of the batten (from top to bottom) seems to have little effect on the wake, neither on the spatial energy distribution nor peak energy. The lower tensioned membrane (left column) resulted in a broader energy distribution and lower intensity peaks. When the applied strain was increased to $8 \%$ (right column), the energy distribution became more focused and the energy intensity increased. Similar to results discussed in the previous paragraph, the fundamental frequency for the higher tensioned membrane did not increase, indicating a relative independence of the free - free configuration from applied and aerodynamic-induced tension.

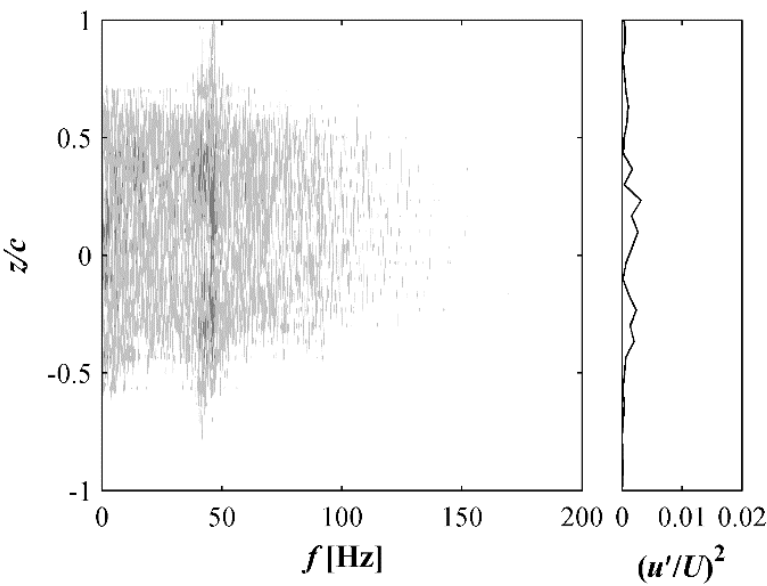

(a) $\varepsilon_{0}=4 \%$, batten at $0.25 c\left(f_{p k}=46 \mathrm{~Hz}\right)$

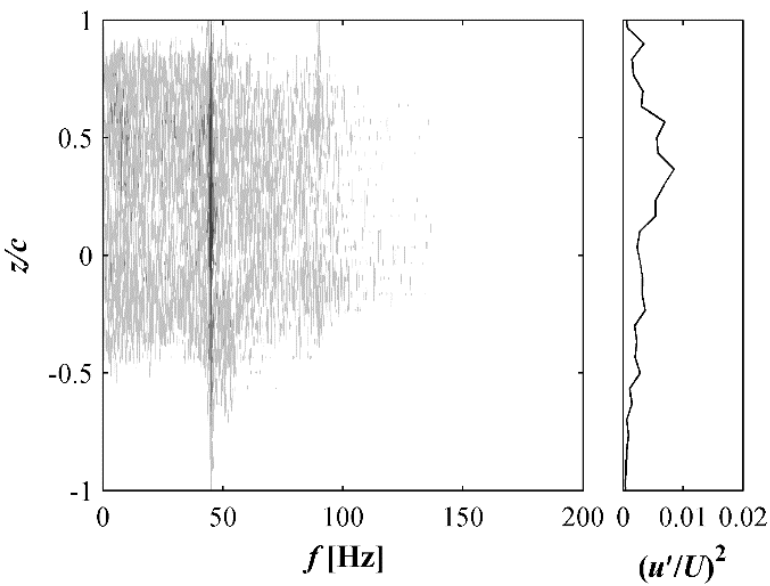

(b) $\varepsilon_{0}=8 \%$, batten at $0.25 c\left(f_{p k}=45 \mathrm{~Hz}\right)$ 

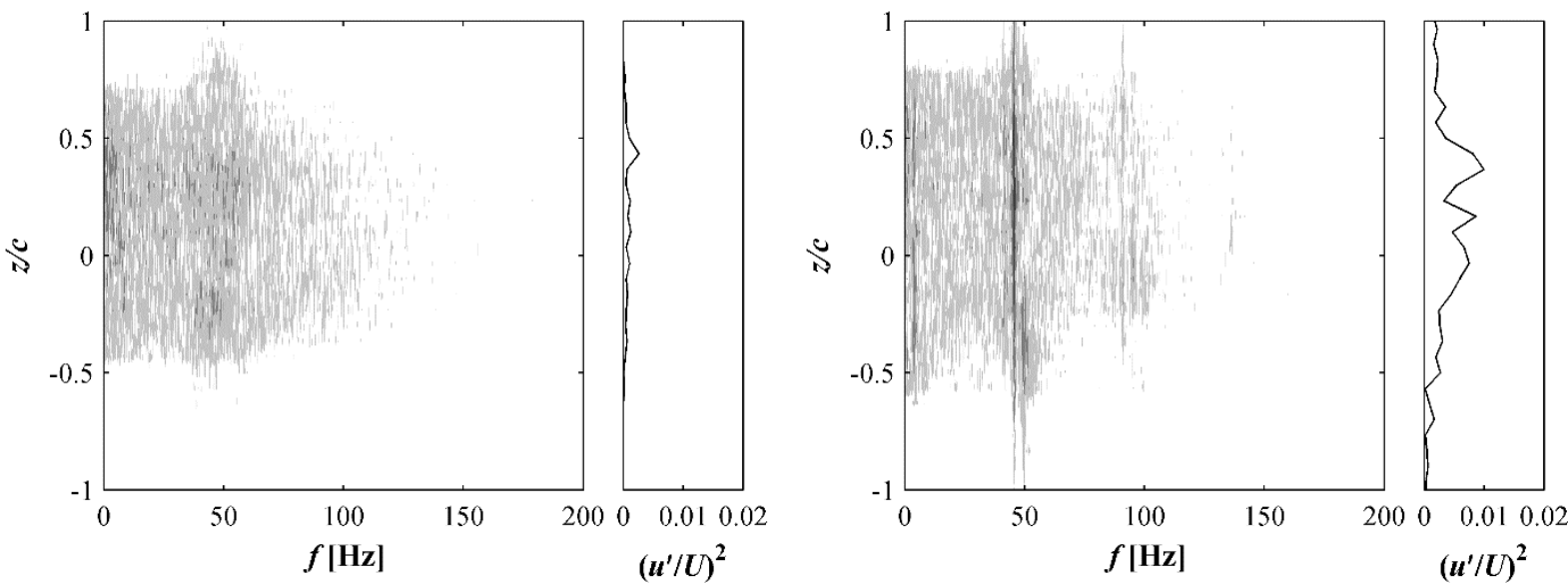

(c) $\varepsilon_{0}=4 \%$, batten at $0.50 \mathrm{c}\left(f_{p k}=54 \mathrm{~Hz}\right)$

(d) $\varepsilon_{0}=8 \%$, batten at $0.50 c\left(f_{p k}=46 \mathrm{~Hz}\right)$
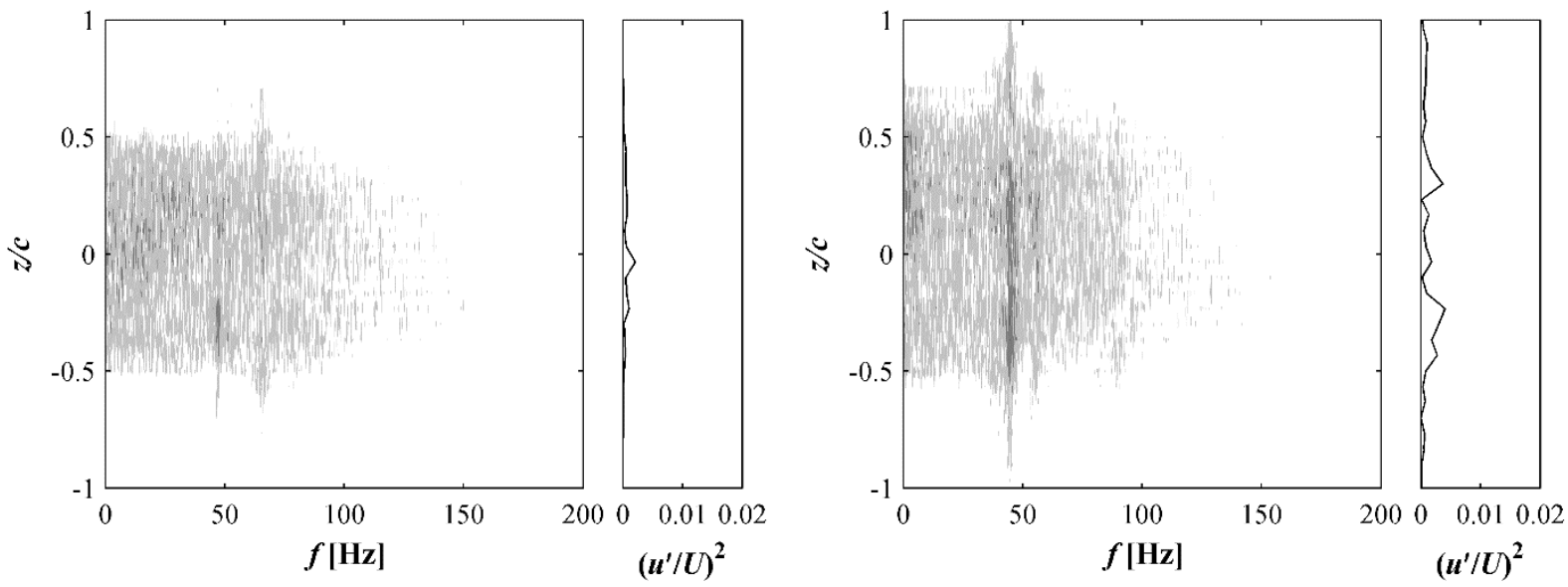

(e) $\varepsilon_{0}=4 \%$, batten at $0.75 c\left(f_{p k}=46 \mathrm{~Hz}\right)$

(f) $\varepsilon_{0}=8 \%$, batten at $0.75 c\left(f_{p k}=45 \mathrm{~Hz}\right)$

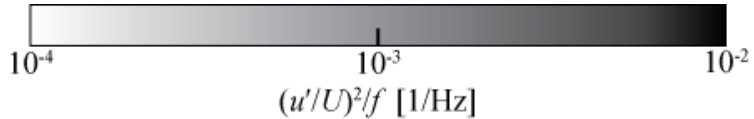

Figure 15 Contour of spectral energy for varying batten position $\left(\alpha=8^{\circ}\right.$ and left: $\Pi_{2}=1.1$, right: $\left.\Pi_{2}=2.2\right)$

\subsection{Aerodynamic Forces}

In this section, the lift coefficient, drag coefficient, and aerodynamic efficiency plots for the spanwise-tensioned membrane cell are presented and discussed. The test schematic is shown in Fig. 1b. The long attachment plates were replaced by the short plates; thus, the aerodynamic forces generated by the membrane were acquired. The LE of the membrane was set as free or fixed, while the TE was free. $\Pi_{2}$ ranged between 0.5 and 2.2 based on the membrane chord length and spanwise tension, and the tests were performed at $R e$ of 55,000. 


\subsubsection{Lift Coefficients}

Figure 16 depicts the lift curve for the membrane cells with a free LE and fixed LE. The applied spanwise strain in the membrane is $4 \%$. At low angles-of-attack, both membranes follow a linear trend up to $8^{\circ}$. The negative zerolift angle of attack for the fixed-free case $(1-0)$ is likely a hysteresis effect of the increasing $\alpha$ trend from negative to positive of the test. As shown in Fig. 17, the lift-curve slope of the fixed-LE, 4\% pre-tensioned membrane is 5.1, nearly identical with that of the solid plate (dash-line in Fig. 17). However, the free-LE membrane shows a higher lift-curve slope than the fixed-LE counterparts at all tested applied strain levels. As the applied strain is increased, the difference in the lift-curve slope between the free- and fixed-LE membranes diminishes. Pre-tensioning the membrane increases the membrane stiffness and reduces the amplitude of the membrane oscillation for both configurations. As a result, when the applied strain was increased from $2 \%$ to $8 \%$, the difference in the lift-curve slope between the fixed-LE and free-LE membranes reduced from $22 \%$ to $3 \%$.

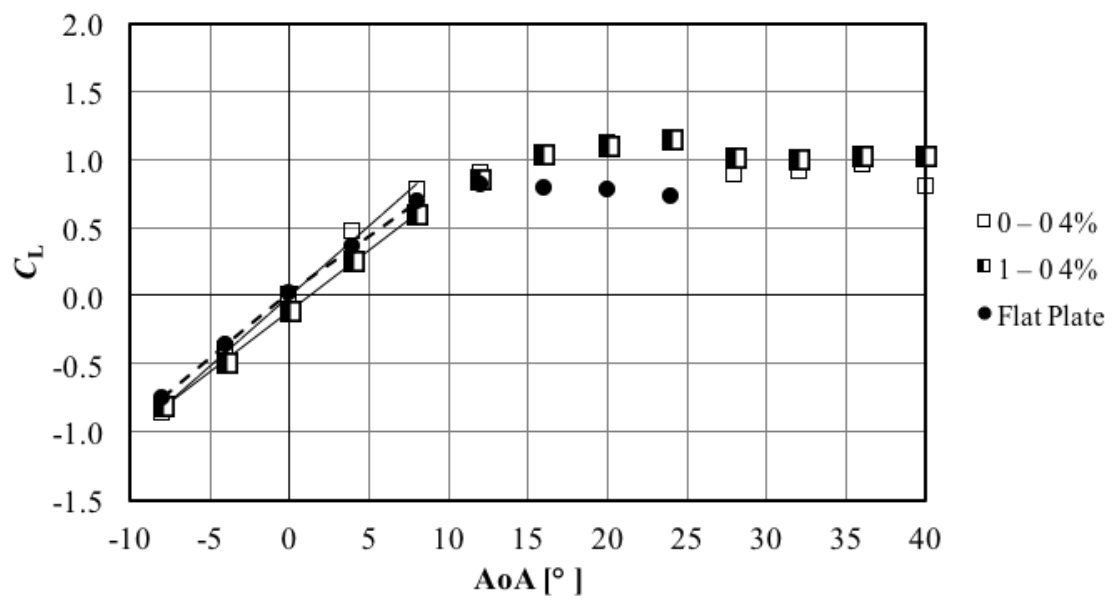

Figure 16 Lift curve for the membrane without and with LE constraint $\left(\varepsilon_{0}=4 \%, \Pi_{2}=1.1\right)$ 


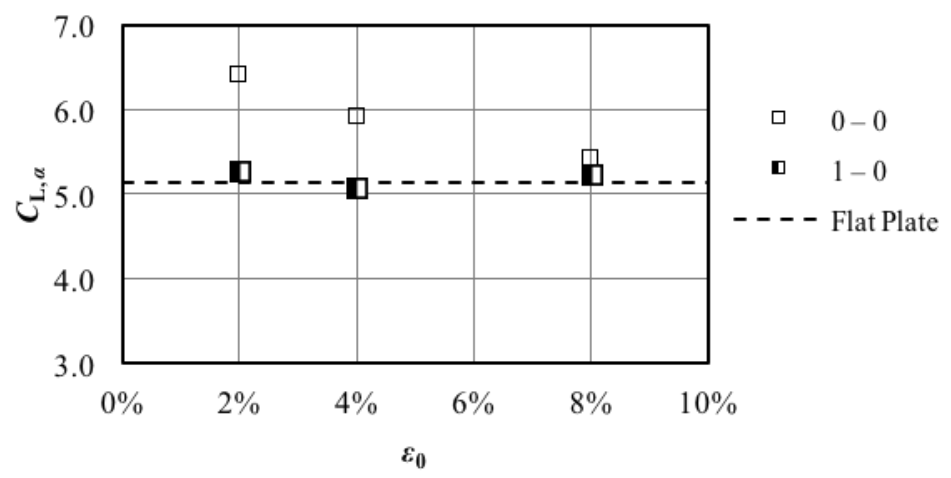

Figure 17 Lift-curve slope for the free and fixed-LE membranes with various applied strain level

As shown in Fig. 16, at higher angles-of-attack $\left(12^{\circ}<\alpha<24^{\circ}\right)$, the free-LE membrane has a similar lift coefficient trend as the fixed-LE membrane. For both membrane specimens, the lift-curve slope decreases relative to the lower angle-of-attack range, showing the onset of stall, and both outperform the flat plate. Measured lift peaks are both at $\alpha=24^{\circ}$. The maximum lift coefficients are plotted in Fig. 18. All strain and both LE configurations exhibited significantly higher peak lift coefficients than the rigid flat plate. The difference between the free-LE and fixed-LE membranes is not significant considering the measurement uncertainty of the lift coefficient which is 0.014 at high angles of attack.

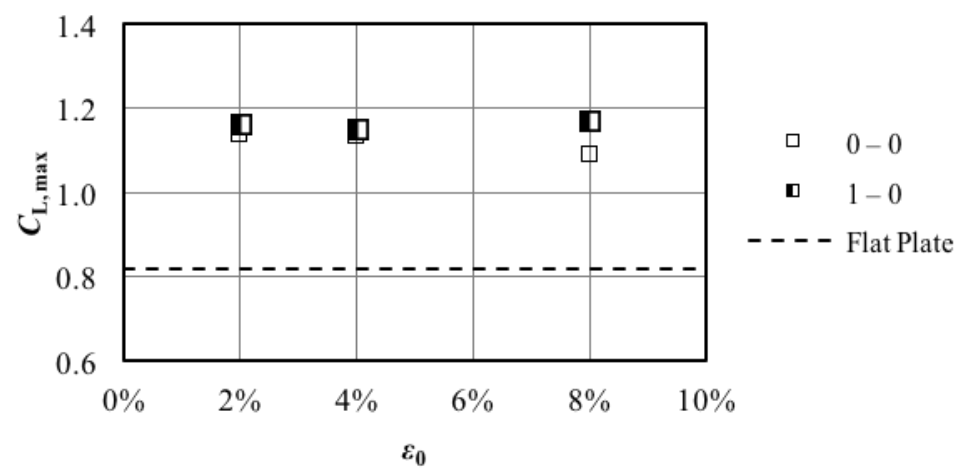

Figure 18 Maximum lift coefficient for the membrane cells with and without LE constraint at various applied strain level 
Figure 19 compares the lift coefficient fluctuation, $C_{\mathrm{L} r m s}{ }^{\prime}$, for the free - free and fixed - free membranes over the angle-of-attack range at $R e=55,000$. The test specimens and conditions are the same as Fig. 16. The free-LE membrane shows a greater fluctuation in the lift coefficient than the fixed-LE membrane at all angles-of-attack. Because the aerodynamic force fluctuation is highly correlated to the membrane instantaneous deflection (Bleischwitz et al., 2015), the higher lift coefficient fluctuation for the free - free configuration likely corresponds to a stronger membrane flutter due to lack of the LE constraint. Note that at the post-stall region $\left(\alpha>24^{\circ}\right)$, both membranes show a decrease in lift coefficient fluctuation, and the lift fluctuation is relatively independent of angleof-attack for both membranes. At the high angles-of-attack, the bluff-body shedding for the membrane wing begins to play a role; thus, the membrane motion tends to be steady (Timpe et al., 2013).

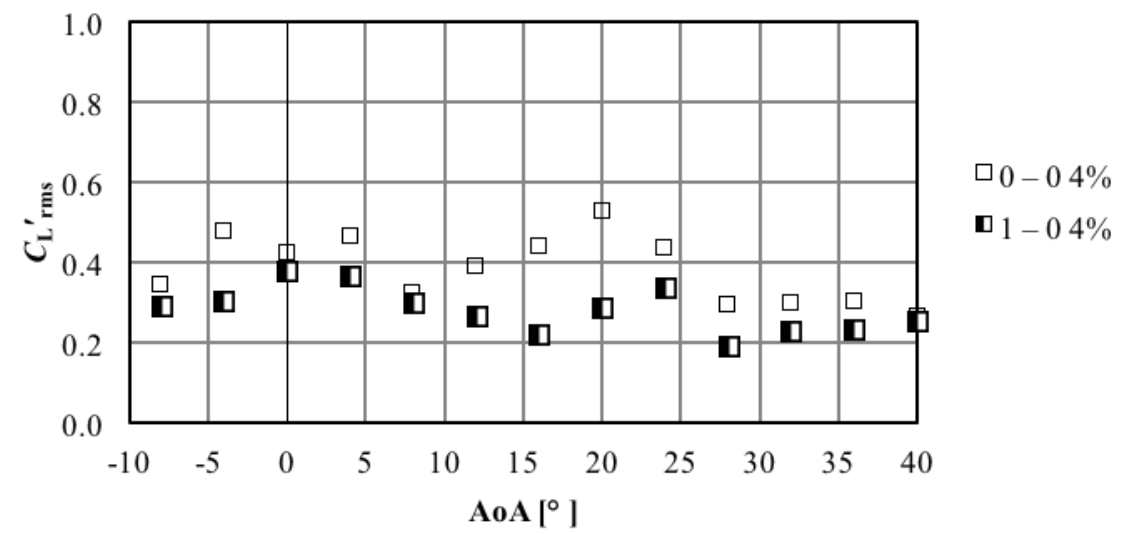

Figure 19 Lift coefficient fluctuation for the membrane cells with and without $\mathrm{LE}$ constraint $\left(\varepsilon_{0}=4 \%, \Pi_{2}=\right.$ 1.1)

\subsubsection{Drag Coefficients}

Figure 20 presents a typical drag plot for the free - free and fixed - free membranes with $\varepsilon_{0}=4 \%$ at $R e=55,000$. The test article and conditions are the same as the case shown in Fig. 16. For the data between $\alpha=-8^{\circ}$ and $8^{\circ}$, the fitted drag coefficient curve was

$$
C_{D}=C_{D, 0}+k\left(\alpha-\alpha_{0}\right)^{2}
$$


where $k$ is a simplified and combined effect of angle-dependent form and induced drag components for a symmetric airfoil, and $\alpha_{0}$ is the zero-lift angle-of-attack. The drag coefficient of the free - free configuration is the greatest among the three test specimens, especially in the pre-stall region. Before the peak lift, LE vibration induced a noticeable increase in the drag coefficient compared with the fixed-LE membrane and flat plate. The fitted minimum drag coefficients of the free - free and fixed - free membranes are 5.1 and 2.6 times higher than the flat plate, respectively. The negative effect on the drag was also witnessed in the wake results, which was presented at Section 4.1. In the post-stall region $\left(>24^{\circ}\right)$, the difference in drag between the free- and fixed-LE membranes diminished as the membrane cell acted more as a bluff body. At higher angles-of-attack, the effect of LE vibration becomes less pronounced. Hubner and Hicks (2011) scalloped the membrane TE of the membrane cell span to effectively reduce the vibration induced drag and retain the benefit of the membrane vibration on lift augmentation. A similar modification at the free LE of the membrane may also eliminate excessive LE oscillation, thus, reducing the drag penalty of the free-LE membrane.

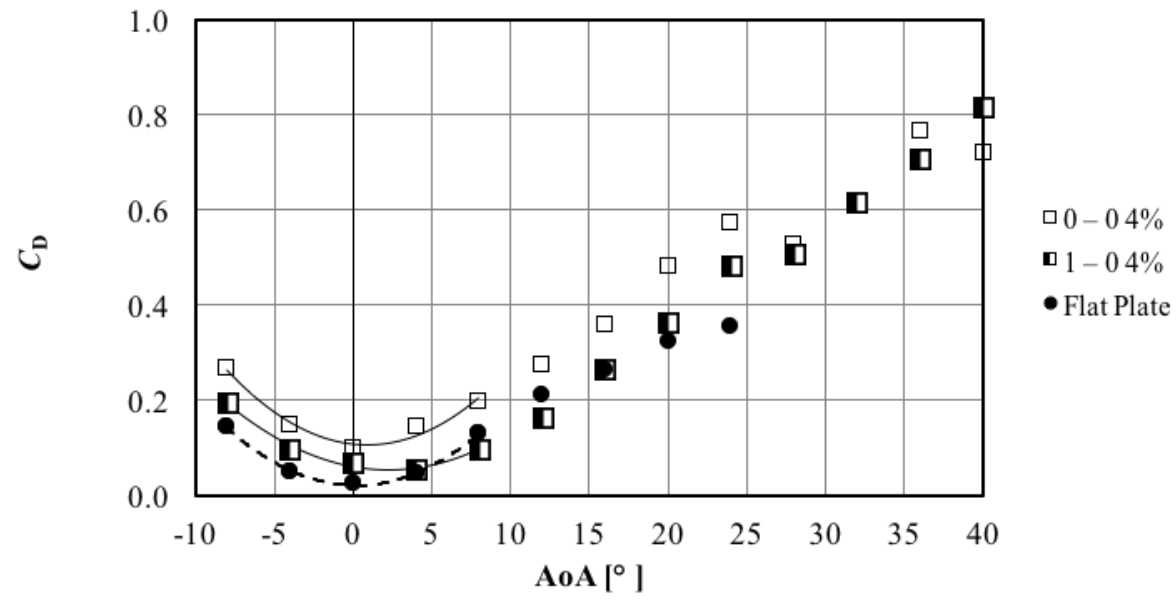

Figure 20 Drag curve for the membrane without and with $\mathrm{LE}$ constraint $\left(\varepsilon_{0}=4 \%, \Pi_{2}=1.1\right)$

The relation between the measured drag coefficient and the wake coefficient for the membrane with the free free and fixed - free configuration is shown in Fig. 21. The plot includes angle-of-attack ( $4^{\circ}$ to $\left.16^{\circ}\right)$ and applied strain $(2 \%, 4 \%$, and $8 \%)$ data. The wake coefficient estimated by the single-sensor hot-wire data is proportional to the membrane drag coefficient with an $R^{2}$ value of 0.95. As discussed in sections 3.2 and 4.1.1, lower values for the wake deficit measurement could be attributed to the lack of velocity decoupling (single wire, oriented sensitive to 
streamwise and vertical components), instantaneous flow reversal (probe placement in region where time-averaged flow reversal was not expected), and streamwise pressure loss (measured less than 1\%). Despite neglecting these corrections, the measured wake coefficient tracks the more accurate drag coefficient measurement fairly well and is useful in comparing the combined effects of wake depth and width as discussed in Section 4.1.2.

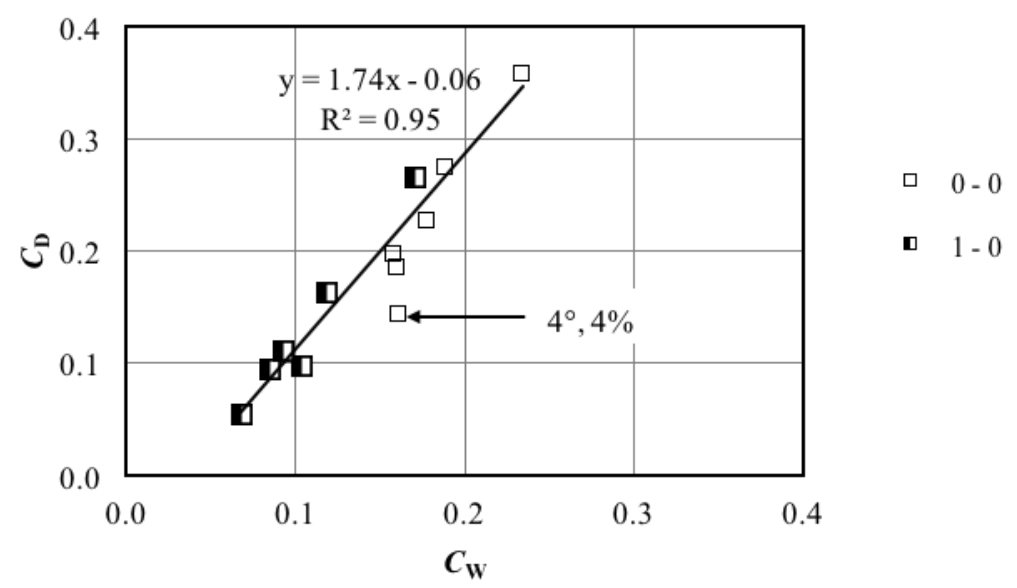

Figure 21 Comparison between drag coefficient and nondimensional momentum loss for the free - free and fixed - free configurations

\subsubsection{Aerodynamic Efficiency}

Figure 22 presents the aerodynamic efficiency trend with respect to the LE condition for the membrane cell at $R e$ $=55,000$. The peak of the aerodynamic efficiency occurred below $\alpha=8^{\circ}$ for all the test specimens. As presented above, the LE vibration generated more lift in the range of $\alpha<8^{\circ}$ but also induced at least twice the drag. Thus, the drag plays a greater role, lowering the aerodynamic efficiency at low angles-of-attack range. The LE vibration deteriorated the aerodynamic efficiency, resulting in a $34 \%$ decrease compared to the fixed - free membrane for the case presented in Fig. 22. The peak efficiency for the rigid flat plate is 7.7, which is superior to all of the membrane configurations regardless of the LE condition or the applied strain level. This is due to the lower drag penalty for the rigid flat plate at low angles-of-attack. At first, the lower aerodynamic efficiency for the membrane cell compared to the rigid plate appears to contradict previous investigations (Timpe et al., 2013; Zhang et al., 2015) where membrane wings outperformed rigid wings. However, these referenced tests focused on low-to-medium aspect ratio, finite-span 
wings (substantial 3D effects); the lift coefficient decreases and the induced drag coefficient increases as aspect ratio decreases. Low aspect ratio trends have a greater effect on rigid wings, resulting in superior results for membrane wings when tip effects are present. In this investigation, finite-span effects were minimized. Beyond the linear lift angle-of-attack range $\left(\alpha>8^{\circ}\right)$ and up to peak lift coefficient $\left(\alpha=24^{\circ}\right)$, the fixed - free membrane cell consistently shows a higher aerodynamic efficiency than the free - free configuration and rigid flat airfoil, exhibiting a better aerodynamic performance at higher angle-of-attack.

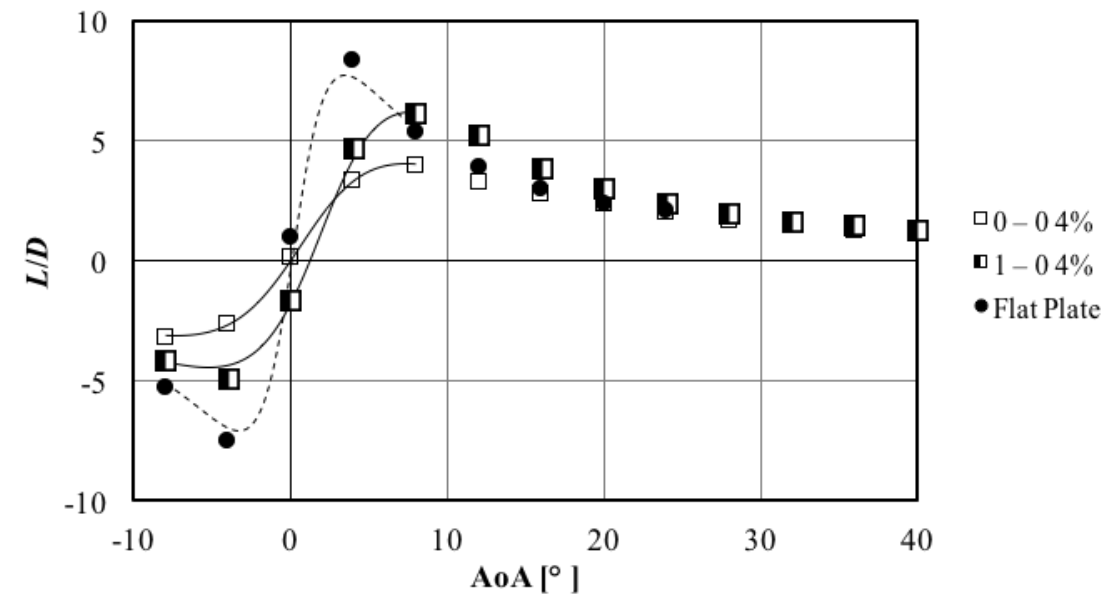

Figure 22 Aerodynamic efficiency for the membranes with and without $L E$ constraint $\left(\varepsilon_{0}=4 \%, \Pi_{2}=1.1\right)$

Figure 23 shows the maximum aerodynamic efficiency with respect to the applied strain for the free - free and fixed - free configurations. The peak efficiency shows a decreasing trend with increasing applied strain for both LE conditions, which is consistent with the finite-span membrane wings discussed in (Zhang et al., 2015). The decrease in the efficiency is attributed to the lower lift coefficient near $\alpha=8^{\circ}$ caused by the higher applied strain level. The fixed - free membrane consistently exhibits a higher aerodynamic efficiency than the free - free membrane due to the lower drag coefficient. On average, LE vibration resulted in a decrease of $28 \%$ in peak aerodynamic efficiency. 


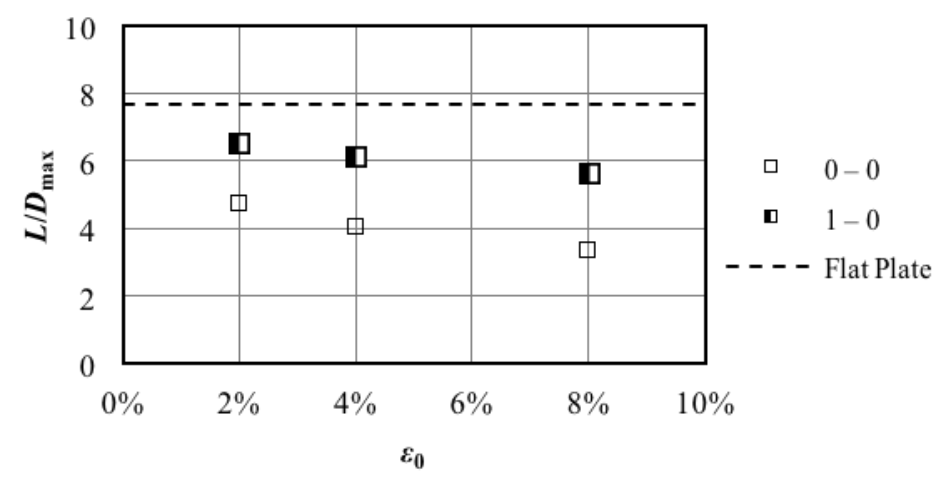

Figure 23 Maximum aerodynamic efficiency for the membrane cells with and without LE constraint

\section{Conclusion}

This paper investigates the LE-vibration effects on a tip-bounded, spanwise-tensioned membrane cell with an aspect ratio of unity in low $R e$ flow. The membrane was mechanically tensioned at $2 \%, 4 \%$, and $8 \%$ in spanwise direction. The aerodynamic tensioning was changed by varying the angle-of-attack ( $4^{\circ}$ to $16^{\circ}$ for wake scan and $-8^{\circ}$ to $40^{\circ}$ for aerodynamic forces measurement) and the dynamic pressure from $40 \mathrm{~Pa}$ to $110 \mathrm{~Pa}$ (most tests were performed at $70 \mathrm{~Pa}$, corresponding to $R e$ of 55,000$)$. The centerline wake was acquired by a single-sensor hot-wire anemometer scanned one chord length aft of the trailing edge of the membrane. The aerodynamic forces on the membrane cell with a free/fixed LE were measured using a dual load-cell setup. By analyzing the time-averaged aerodynamic forces, wake mean and rms velocity profiles, and corresponding power spectra, the following conclusions are made:

1. Membranes with a free (unconstrained) LE did not exhibit better aerodynamic characteristics than the fixed (constrained) LE counterparts. The oscillation of the membrane did not generate thrust nor reduce the drag. Although the lift coefficient at the pre-stall angle was increased by the free LE, the LE vibration caused increased drag at the pre-stall region, thus, resulting in a lower aerodynamic efficiency.

2. Relative to a membrane constrained at the LE and TE, an unconstrained LE with either free or fixed TE increases the wake width (mixing), wake coefficient (momentum loss), and turbulence intensity in the wake. The wake characteristics of a membrane with an unconstrained LE are less affected by the angle-of-attack and 
applied strain. The energy spectra are broader and less organized. This is a result of the large amplitude vibration of the LE, which acted to reduce the effective membrane stiffness.

3. Relative to a membrane constrained at the LE and TE, an unconstrained TE with either free or fixed LE decreases the wake depth but also increases the momentum loss; however, for the fixed - free configuration, the momentum loss is not large. The wake characteristics are affected by the angle-of-attack but not significantly by the applied strain. The energy spectra are more organized for the fixed-LE configuration, clearly depicting a fundamental frequency, bimodal distribution and the highest spectral energy peak. These traits indicate a more coherent fluid-structure interaction.

4. The interior spanwise constraints applied to a free - free configuration at $25 \%, 50 \%$, and $75 \%$ of the chord have little influence on the wake characteristics compared to the free - fixed configuration. Once the LE is free to vibrate, the spanwise constraints play an insignificant role on wake characteristics and energy spectra.

5. Adding a LE constraint increases the fundamental frequency of vibration whereas the spanwise constraints further downstream, including the TE, has little effect on the fundamental frequency. The LE constraint effectively enables increased aerodynamic tensioning.

\section{Acknowledgments}

The research is supported through Air Force Office of Scientific Research (AFOSR) through the grant FA955010-1-0152, under a program managed by Dr. Douglas Smith. The authors also would like to thank support from the National Science Foundation REU program for Mr. Wrist, through the grant EEC-1062611.

\section{References}

Abudaram, Y., Ifju, P.G., Hubner, J.P., Ukeiley, L., 2014. Controlling Pretension of Silicone Membranes on Micro Air Vehicle Wing. The Journal of Strain Analysis for Engineering Design 49, 161-170.

Albertani, R., Stanford, B., Hubner, J.P., Ifju, P.G., 2007. Aerodynamic coefficients and deformation measurements on flexible micro air vehicle wings. Exp Mech 47, 625-635.

Arbós-Torrent, S., Ganapathisubramani, B., Palacios, R., 2013. Leading- and trailing-edge effects on the aeromechanics of membrane aerofoils. Journal of Fluids and Structures 38, 107-126. 
Bleischwitz, R., Kat, R.d., Ganapathisubramani, B., 2015. Aspect-Ratio Effects on Aeromechanics of Membrane Wings at Moderate Reynolds Numbers. AIAA Journal 53, 780-788.

Burns, T., Mueller, T.J., 1982. Experimental Studies of the Eppler 61 Airfoil at Low Reynolds Numbers, 20th AIAA Aerospace Sciences Meeting. American Institute of Aeronautics and Astronautics, Orlando, Florida.

Hu, H., Tamai, M., Murphy, J.T., 2008. Flexible-Membrane Airfoils at Low Reynolds Numbers. Journal of Aircraft 45, 17671778.

Hubner, J.P., Hicks, T., 2011. Trailing-edge scalloping effect on flat-plate membrane wing performance. Aerosp Sci Technol 15, $670-680$.

Jaworski, J.W., 2012. Thrust and Aerodynamic Forces from an Oscillating Leading Edge Flap. AIAA Journal 50, $2928-2931$.

Jones, A.R., Babinsky, H., 2008. Low Reynolds Number Aerodynamics of Leading-Edge Flaps. Journal of Aircraft 45, $342-345$.

Laitone, E.V., 1997. Wind tunnel tests of wings at Reynolds numbers below 70 000. Experiments in Fluids 23, 405-409.

Lissaman, P.B.S., 1983. Low-Reynolds-number airfoils. Annual Review of Fluid Mechanics 15, 223-239.

Mueller, T.J., 1985. The influence of laminar separation and transition on low Reynolds number airfoil hysteresis. Journal of Aircraft 22, 763-770.

Mueller, T.J., 1999. Aerodynamic Measurements at Low Reynolds Numbers for Fixed Wing Micro-Air Vehicles, RTO AVT/VKI Special Course.

Mueller, T.J., DeLaurier, J.D., 2003. Aerodynamics of Small Vehicles. Annual Review of Fluid Mechanics 35, 89-111.

Mueller, T.J., Kellogg, J.C., Ifju, P., Shkarayev, S.V., 2007. Introduction to the Design of Fixed-Wing Micro Air Vehicles: Including Three Case Studies. AIAA, Reston, VA.

Rojratsirikul, P., Wang, Z., Gursul, I., 2010. Effect of pre-strain and excess length on unsteady fluid-structure interactions of membrane airfoils. Journal of Fluids and Structures 26, 359-376.

Scott, K.D., Hubner, J.P., Ukeiley, L., 2012. Cell Geometry and Material Property Effects on Membrane and Flow Response. AIAA Journal 50, 755-761. 
Shyy, W., Lian, Y., Tang, J., Viieru, D., Liu, H., 2007. Aerodynamics of Low Reynolds Number Flyers. Cambridge University Press, Cambridge.

Stanford, B., Ifju, P., 2008. The Validity Range of Low Fidelity Structural Membrane Models. Exp Mech 48, 697-711.

Timpe, A., Zhang, Z., Hubner, J., Ukeiley, L., 2013. Passive flow control by membrane wings for aerodynamic benefit. Experiments in Fluids 54.

Triantafyllou, M.S., Triantafyllou, G.S., Gopalkrishnan, R., 1991. Wake Mechanics for Thrust Generation in Oscllating Foils. Physics of Fluids A 3, 2835-2838.

Zhang, Z., Wrist, A., Hubner, J.P., Ukeiley, L., 2014. Wake Characteristics of a 2D Spanwise Tensioned Membrane with Aerodynamic Loading, 52nd AIAA Aerospace Sciences Meeting. American Institute of Aeronautics and Astronautics.

Zhang, Z., Hopper, L., Wrist, A., Hubner, J.P., Ukeiley, L., 2014b. Nondimensional Frequency Scaling of Aerodynamicallytensioned Membranes. Journal of Fluids and Structures 48, 14-26.

Zhang, Z., Martin, N., Wrist, A., Hubner, J.P., 2015. Geometry and Prestrain Effects on the Aerodynamic Characteristics of Batten-Reinforced Membrane Wings. Journal of Aircraft 53, 530-544. 